\title{
A Coupled Aeroelastic Model for Launch Vehicle Stability Analysis
}

\author{
Jeb S. Orr ${ }^{*}$ \\ Science Applications International Corporation, Huntsville, AL 35802
}

\begin{abstract}
[Abstract] A technique for incorporating distributed aerodynamic normal forces and aeroelastic coupling effects into a stability analysis model of a launch vehicle is presented. The formulation augments the linear state-space launch vehicle plant dynamics that are compactly derived as a system of coupled linear differential equations representing small angular and translational perturbations of the rigid body, nozzle, and sloshing propellant coupled with normal vibration of a set of orthogonal modes. The interaction of generalized forces due to aeroelastic coupling and thrust can be expressed as a set of augmenting non-diagonal stiffness and damping matrices in modal coordinates with no penalty on system order. While the eigenvalues of the structural response in the presence of thrust and aeroelastic forcing can be predicted at a given flight condition independent of the remaining degrees of freedom, the coupled model provides confidence in closed-loop stability in the presence of rigid-body, slosh, and actuator dynamics. Simulation results are presented that characterize the coupled dynamic response of the Ares I launch vehicle and the impact of aeroelasticity on control system stability margins.
\end{abstract}

\begin{tabular}{|c|c|c|c|}
\hline \multirow[b]{2}{*}{$V$} & \multicolumn{3}{|c|}{ Notation } \\
\hline & $\begin{array}{l}\text { Velocity magnitude with respect to Earth-centered } \\
\text { inertial frame }\end{array}$ & $z$ & Translational perturbation, trajectory frame \\
\hline$M$ & Vehicle total mass & $\varphi$ & Angular perturbation, trajectory frame \\
\hline $\bar{g}$ & Acceleration along $x^{B}$ axis, $(F-D) / M$ & $\eta_{i}$ & State of $i^{\text {th }}$ elastic eigenmode \\
\hline$F, R$ & Total thrust force and gimbaled thrust force & $\bar{q}$ & Dynamic pressure \\
\hline$\alpha$ & Effective angle of attack & $D$ & Drag force, $q A C_{A}$ \\
\hline$A$ & Cross section reference area & $S$ & Span reference area \\
\hline$I$ & Total vehicle moment of inertia about $\mathrm{CM}$ & $l_{x}$ & Aerodynamic moment arm of $x^{\text {th }}$ station \\
\hline$\left[\frac{\partial C_{N}}{\partial \alpha}\right]_{x}$ & $\begin{array}{l}\text { Linearized aerodynamic partial derivative of normal } \\
\text { force coefficient at } x^{\text {th }} \text { station }\end{array}$ & $C_{A}$ & Axial drag force coefficient \\
\hline & Generalized mass, $i^{\text {th }}$ elastic eigenmode & $\psi_{g i}, \psi_{g i}^{\prime}$ & Mode shape and slope of $i^{\text {th }}$ mode at gimbal \\
\hline$\omega_{b i}, \zeta_{b i}$ & $\begin{array}{l}\text { Natural frequency and damping ratio of } i^{\text {th }} \text { bending } \\
\text { mode }\end{array}$ & $\psi_{i x}, \dot{\psi}_{i x}$ & Mode shape and slope of $i^{\text {th }}$ mode at $x^{\text {th }}$ station \\
\hline
\end{tabular}

\section{Introduction}

A well-known technique for assessing the short-period dynamic stability of an aircraft or missile in atmospheric flight involves the use of a set of linearized equations of motion about some trimmed or nominal operating condition. For a large, flexible launch vehicle, the problem is less trivial in that many additional degrees of freedom must be considered to effect proper stabilization of the actual plant dynamics. Due to their necessarily low structural mass, large boosters are very flexible; sensed angles and rates are easily corrupted by vibrations and significant dynamic coupling can occur among the structural modes, propellant slosh modes, and actuator dynamics.

* Flight Controls Engineer, Advanced Technology Division, jeb.orr@nasa.gov 
Recent work has been undertaken ${ }^{5,6}$ to revisit the assumptions of the launch vehicle perturbation models ${ }^{1-4}$ that have been used in control design studies since the beginning of the ballistic missile era. A traditional technique is a careful elimination of the dynamic coupling paths that are deemed negligible or too difficult to handle analytically via transfer function methods. ${ }^{1}$ The advent of rapid matrix-based numerical computation and a state-space implementation of the equations of motion allow the construction of a high-fidelity, high-order linear model, the response of which can be readily assessed for the purposes of stability analysis and control design. ${ }^{6}$ Such a model is dynamically complete in that all of the terms evolved from the linear expression of the kinetic and potential energies are accounted for in the coupled dynamics; that is, the system should conserve energy when integrated numerically in the absence of external forces. Conveniently, complex elastic cross-coupling effects that were intractable to implement on analog computers can now be incorporated into the model via elegant linear algebra operations. For typical launch vehicles that by design operate in a linear regime, a linear stability analysis model can accurately capture the actual physics without resorting to a complex and computationally intensive multibody/multiphysics simulation approach.

The present work extends the previous results by investigating the effects of additional generalized forces due to coupling of distributed aerodynamic loads and lateral bending of the vehicle structure and illustrates a technique for incorporating the relevant expressions into the previously derived state-space dynamics model. While similar solutions for aircraft structures are well known in the aeroelastic community, a straightforward technique for quantifying impacts to closed-loop control of launch vehicles in the presence of a thrust force and additional degrees of freedom does not appear to be readily available. Since the thrust vector control (TVC) loop bandwidth is limited and the nozzle is commanded with respect to the thrust structure, thrust forces (follower forces) rotate and translate in inertial space due to vehicle bending and also affect the bending dynamics. Although the rigid-body dynamics may be unstable in the absence of closed-loop attitude control, the dynamic stability of the open-loop system in the presence of aerodynamic and thrust forces can also be investigated in order to quantify the conservatism of other tools (e.g., nonlinear simulations) for which the implementation of aeroelastic models is excessively complex or computationally prohibitive.

\section{Local Angles of Attack}

As discussed in [6], the coupled plant dynamics are desired in an explicit state-space form suitable for numerical integration or stability analysis; the system

$$
\dot{x}=\underbrace{E^{-1} A}_{\mathscr{A}} x+\underbrace{E^{-1} B}_{\tilde{B}} u
$$

can be obtained from the system matrices $\tilde{\boldsymbol{A}}, \tilde{\boldsymbol{B}}$ via an inversion after writing the system dynamics in the general descriptor form as

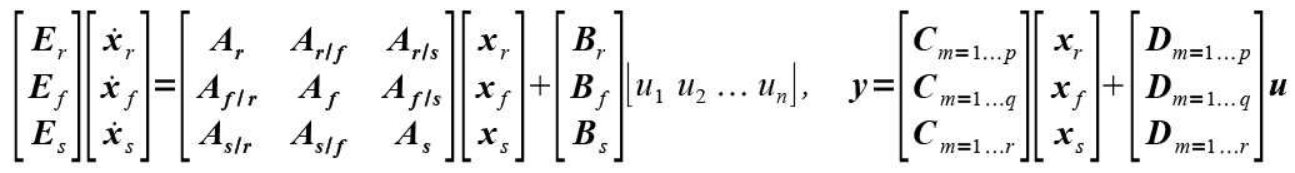

using the block diagonal approach, with the state vector

$$
\boldsymbol{x}=\left[\begin{array}{lllllllllllllllllll}
\varphi & \dot{\varphi} & z & \eta_{1} & \eta_{2} & \ldots & \eta_{k} & \dot{\eta}_{1} & \dot{\eta}_{2} & \ldots & \dot{\eta}_{k} & \delta_{s 1} & \delta_{s 2} & \ldots & \delta_{s n} & \dot{\delta}_{s 1} & \dot{\delta}_{s 2} & \ldots & \dot{\delta}_{s n}
\end{array}\right]^{T} .
$$

Here, $\boldsymbol{A}_{\boldsymbol{r}}$ represents the rigid-body dynamics in the pitch (yaw) plane, $\boldsymbol{A}_{\boldsymbol{f}}$ includes the diagonal matrix of eigenvalues and structural damping coefficients used to construct the homogeneous flex dynamics, and $\boldsymbol{A}_{s}$ contains the slosh dynamics. We will use the approach of augmenting the $\boldsymbol{A}_{\boldsymbol{r}}$ and $\boldsymbol{A}_{\boldsymbol{f}}$ dynamics with the components of rigid and elastic angle of attack so as to capture both pitch damping and structural interaction due to distributed aerodynamics, ${ }^{2}$ and the relevant input matrices $\boldsymbol{B}_{r}, \boldsymbol{B}_{f}$ will be modified to include wind gust effects.

In most missile stability analysis models appearing in the literature, aerodynamics effects are accounted for via rigid body normal force and moment coefficients that are reduced to an equivalent center of pressure and normal force slope with respect to a trajectory-referenced angle of attack (point mass aerodynamics). ${ }^{3}$ The components of the rigid-body angle of attack in the plane of motion are given by small-angle linearization of the rigid-body pitch 
angle and lateral velocity in a quasi-inertial frame which is fixed with respect to the longitudinal axis of the vehicle. At the center of mass, the rigid-body angle of attack is described by

$$
\alpha=\varphi-\frac{\dot{z}}{V}+\frac{w}{V},
$$

where $\varphi$ is the trajectory-referenced rigid-body perturbation angle, $\dot{z}$ is the lateral velocity normal to the trajectory, and $w$ is the rigid body gust velocity. ${ }^{3} \mathrm{~A}$ small angle linearization of the inverse tangent has been used, with $\dot{z}, w \ll V$. These assumptions are reasonable for a typical booster vehicle on a gravity turn trajectory.

If the angle of attack is computed at a station on the vehicle other than at the center of mass, the rigid translational velocity relative to the freestream and the angular displacement affect the angle of attack, and the gust velocity is a local velocity. Local angular displacements and lateral velocities consist of the sum of rigid body motion and flexibility effects.

The local angular displacement in the pitch plane of the $x^{\text {th }}$ discrete section of the vehicle with respect to the freestream velocity, with $x$ an integer varying from 1 to $l$, is given by

$$
\alpha_{x}=\varphi-\frac{\dot{z}}{V}-\frac{l_{x}}{V} \dot{\varphi}+\frac{w_{x}}{V}+\sum_{i=1}^{k}\left(\dot{\psi}_{i x}^{\prime} \eta_{i}-\frac{1}{V} \psi_{i x} \dot{\eta}_{i}\right) .
$$

The local translational velocity of the $x^{\text {th }}$ station is the sum of the translational velocity of the center of mass, the translational velocity due to the rigid angular rate, and the elastic velocity of the associated node. ${ }^{2}$ These terms comprise those with coefficients of $V^{-1}$, the latter two of which are sometimes neglected in stability studies. The components of the local angle of attack are illustrated in Figure 1.

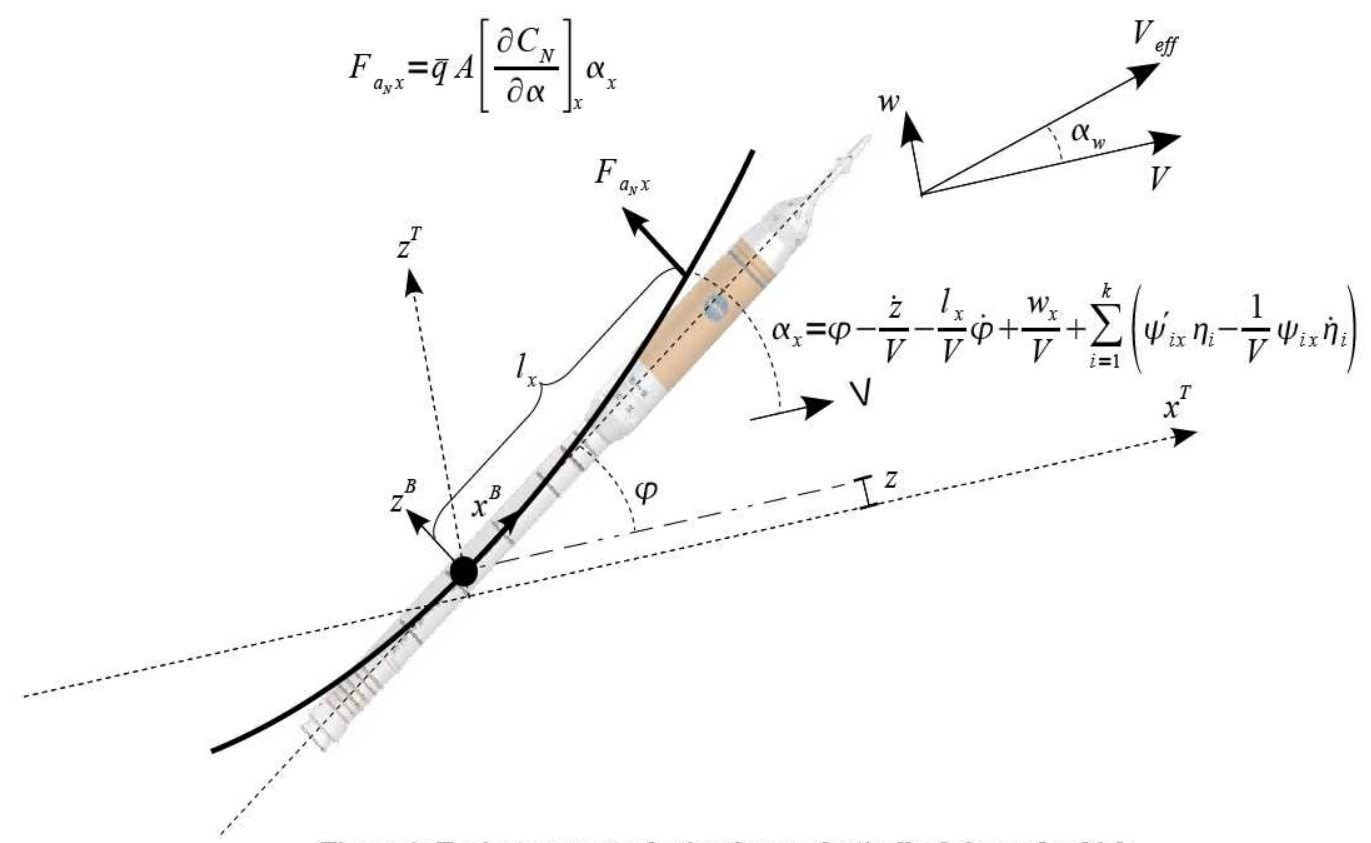

Figure 1: Trajectory perturbation for an elastically deformed vehicle

Since the linear elasticity model describes motion at distinct gridpoints, the vehicle line load data is converted from a continuous distribution into a set of discrete rigid partial derivatives. For each $x$ station from 1 to $l$, there is a corresponding load vector

$$
\boldsymbol{d}_{\boldsymbol{x}}=\left[\begin{array}{llll}
{\left[\frac{\partial C_{N}}{\partial \alpha}\right]_{1}} & {\left[\frac{\partial C_{N}}{\partial \alpha}\right]_{2}} & \cdots & {\left[\frac{\partial C_{N}}{\partial \alpha}\right]_{l}}
\end{array}\right]^{T}
$$


that is derived from piecewise integration of the continuous distribution. Each local coefficient does not take into account dynamic propagation of flow along the vehicle nor the effects of elastic motion or deformation on this flow; it is purely a static-load, rigid-body assessment of the normal force distribution. This distribution is a first-order discrete approximation of the true dynamic loads under the assumption that deflections are small and do not significantly affect downstream flow. ${ }^{7}$ A typical discrete line load distribution and the continuous data from which it is derived appear in Figure 2.

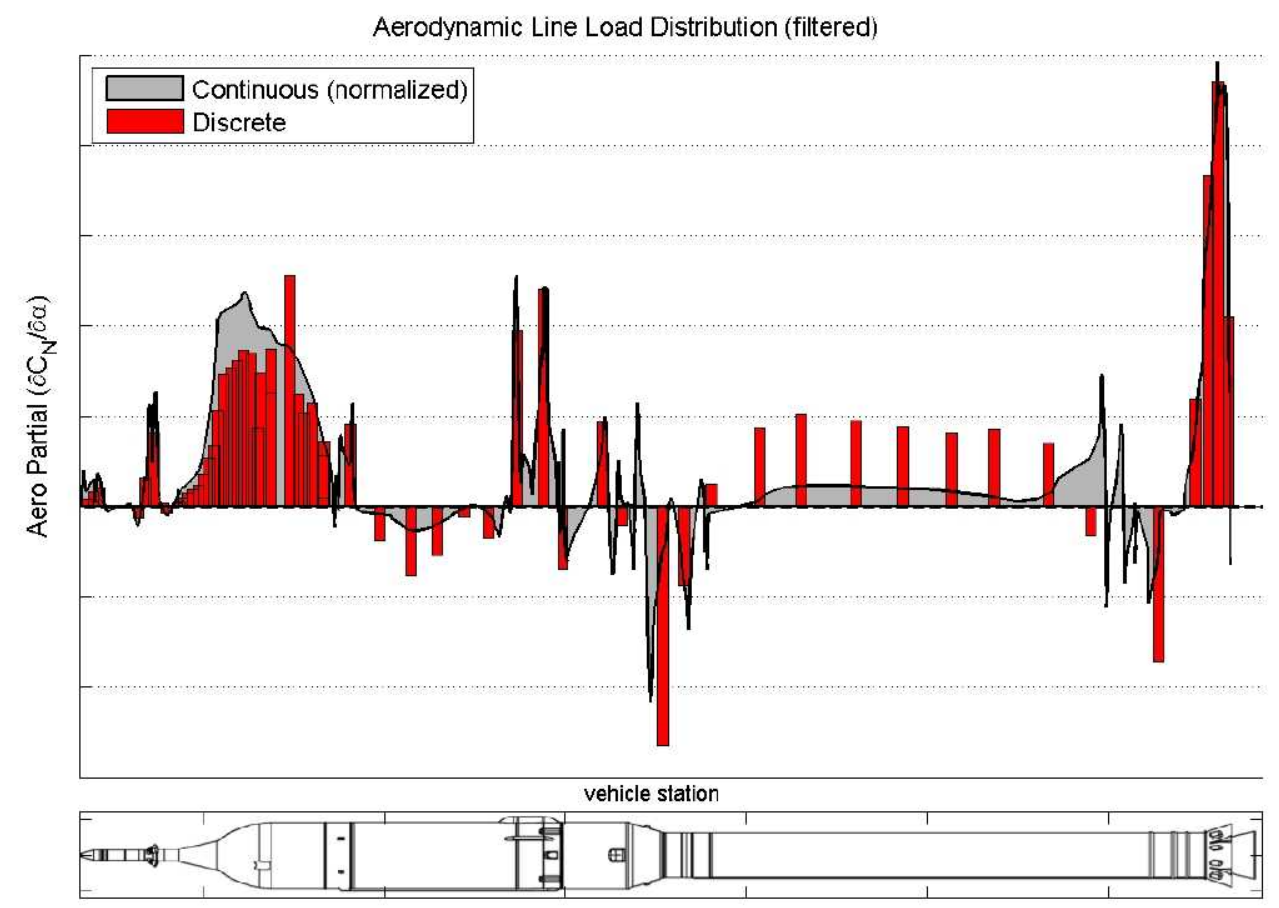

Figure 2: Typical discrete line load distribution

The equations of motion for the flexible vehicle ${ }^{6}$ can be augmented with the complete angle of attack expression in (5). The three fundamental coupled equations (rigid body rotation, rigid body translation, and lateral bending) can be written as

$$
\begin{gathered}
I \ddot{\varphi}=\bar{q} S \sum_{x=1}^{l} l_{x}\left[\frac{\partial C_{N}}{\partial \alpha}\right]_{x}\left(\varphi-\frac{\dot{z}}{V}-\frac{l_{x}}{V} \dot{\varphi}+\frac{w_{x}}{V}+\sum_{i=1}^{k} \psi_{i x}^{\prime} \eta_{i}-\frac{1}{V} \sum_{i=1}^{k} \psi_{i x} \dot{\eta}_{i}\right) \\
M z-M \bar{g} \varphi=\bar{q} S \sum_{x=1}^{l}\left[\frac{\partial C_{N}}{\partial \alpha}\right]_{x}\left(\varphi-\frac{\dot{z}}{V}-\frac{l_{x}}{V} \dot{\varphi}+\frac{w_{x}}{V}+\sum_{i=1}^{k} \psi_{i x}^{\prime} \eta_{i}-\frac{1}{V} \sum_{i=1}^{k} \psi_{i x} \dot{\eta}_{i}\right) \\
\mu_{i}\left(\ddot{\eta}_{i}+2 \zeta_{b i} \omega_{b i} \dot{\eta}+\omega_{b i}^{2} \eta_{i}\right)=\bar{q} S \sum_{x=1}^{l} \psi_{i x}\left[\frac{\partial C_{N}}{\partial \alpha}\right]_{x}\left(\varphi-\frac{\dot{z}}{V}-\frac{l_{x}}{V} \dot{\varphi}+\frac{w_{x}}{V}+\sum_{\rho=1}^{k} \psi_{\rho x}^{\prime} \eta_{\rho}-\frac{1}{V} \sum_{\rho=1}^{k} \psi_{\rho x} \dot{\eta}_{\rho}\right) .
\end{gathered}
$$

Nozzle inertial coupling, slosh dynamics, and thrust forces are included in the model ${ }^{6}$ but not explicitly written here since no direct coupling from aerodynamics occurs via those terms. The right-hand sides of (7), (8), and (9) represent only aerodynamic forces resulting from rigid-body rotation, rigid-body translation, elastic deformation, and wind gusts. 


\section{Rigid Body Aerodynamic Damping}

In [6] it was shown that the rigid-body dynamics could be written as

$$
\boldsymbol{A}_{r}=\left[\begin{array}{ccc}
0 & 1 & 0 \\
-\frac{\partial C_{N}}{\partial \alpha} \bar{q} S l_{a} & 0 & \frac{\partial C_{N}}{\partial \alpha} \frac{\bar{q} S}{V} l_{a} \\
M \bar{g}+\frac{\partial C_{N}}{\partial \alpha} \bar{q} S & 0 & -\frac{\partial C_{N}}{\partial \alpha} \frac{\bar{q} S}{V}
\end{array}\right],
$$

assuming only a rigid-body force and moment partial derivative for $\alpha$ acting at a center of pressure location $l_{a}$.

The requisite rigid coupling matrices must be constructed from the vectors

$$
\boldsymbol{l}_{\boldsymbol{x}}=\left[\begin{array}{llll}
l_{1} & l_{2} & \ldots & l_{l}
\end{array}\right]^{T},
$$

which is an $l \times 1$ column vector of $x$ station displacements and

$$
l_{x}{ }^{2}=\left[\begin{array}{llll}
l_{1}^{2} & l_{2}^{2} & \ldots & l_{l}^{2}
\end{array}\right]^{T},
$$

also an $l \times 1$ column vector. The rigid-body matrices are modified with the equivalent rigid-body partial derivatives computed from (6), (11), and (12), such that

$$
\boldsymbol{A}_{r}=\left[\begin{array}{ccc}
0 & 1 & 0 \\
\bar{q} S k_{r} & -\frac{\bar{q} S \dot{k}_{r}}{V} & -\frac{\bar{q} S k_{r}}{V} \\
M \bar{g}+\bar{q} S k_{t} & -\frac{\bar{q} S k_{r}}{V} & -\frac{\bar{q} S k_{t}}{V}
\end{array}\right],
$$

where $k_{t}$ is the sum of the elements of $\boldsymbol{d}_{\boldsymbol{x}}$, and

$$
k_{r}=\boldsymbol{l}_{x}^{T} \boldsymbol{d}_{\boldsymbol{x}}, \quad \dot{k}_{r}=\left[\boldsymbol{l}_{x}^{2}\right]^{T} \boldsymbol{d}_{\boldsymbol{x}} .
$$

Note the pitch damping terms appearing in (13). An inspection of the state vector (3) reveals damping effects on both the translational and angular accelerations due to angular velocity of the rigid body. The matrix (13) accounts for the first three terms on the right-hand side of (7) and (8).

\section{Aeroelastic - Rigid Dynamic Coupling}

As it will be required shortly, define the binary vector operator

$$
\boldsymbol{a} * \boldsymbol{b}=\left[\begin{array}{llll}
a_{1} b_{1} & a_{2} b_{2} & \ldots & a_{n} b_{n}
\end{array}\right]
$$

where $\boldsymbol{a}, \boldsymbol{b}$ are row or column vectors of the same dimension, and the result is a row or column vector, accordingly, having as its elements the products of the elements of $\boldsymbol{a}, \boldsymbol{b}$. This is equivalent to the ' ${ }^{*}$ ' operation appearing in the matrix programming language $M A T L A B^{\circledR}$ (The MathWorks, Inc.).

Let $\psi_{i x}, \psi_{i x}$ be the mode shape and slope, or the elements of the $i^{\text {th }}$ mode eigenvector at the $x^{\text {th }}$ station. Define the gridpoint influence matrices

$$
\boldsymbol{\Psi}_{a}=\left[\begin{array}{cccc}
\psi_{11} & \psi_{12} & \ldots & \psi_{1 l} \\
\psi_{21} & \psi_{22} & \ldots & \psi_{2 l} \\
\vdots & & & \vdots \\
\psi_{k 1} & \psi_{k 2} & \ldots & \psi_{k l}
\end{array}\right], \quad \boldsymbol{\Psi}_{a}=\left[\begin{array}{cccc}
\psi_{11}^{\prime} & \psi_{12}^{\prime} & \ldots & \psi_{1 l}^{\prime} \\
\psi_{21}^{\prime} & \psi_{22}^{\prime} & \ldots & \dot{\psi}_{2 l}^{\prime} \\
\vdots & & & \vdots \\
\psi_{k 1}^{\prime} & \psi_{k 2}^{\prime} & \ldots & \psi_{k l}^{\prime}
\end{array}\right]
$$

which are each of dimension $k \times l$ over $k$ modes and $l$ stations. The choice of gridpoints to represent the local deformation of the vehicle obviously should well-represent overall motion of the load structure (and hence the outer 
mold line) and not local elasticity. The use of centerline gridpoints in this manner is generally only accurate for low frequency global deformations, such as the first and second bending modes.

Rigid-body coupling from flex occurs due to force follower terms in addition to distributed aerodynamics. The rigid coupling matrix due to elastic force following is already given by ${ }^{\dagger}$

$$
\boldsymbol{A}_{r / f 1}=\left[\begin{array}{cccc|c}
{\left[\mathbf{0}_{1 \times k}\right]} & & \\
-R\left(l_{g} \psi_{g 1}^{\prime}+\psi_{g 1}\right) & -R\left(l_{g} \psi_{g 2}^{\prime}+\psi_{g 2}\right) & \ldots & -R\left(l_{g} \psi_{g k}^{\prime}+\psi_{g k}\right) & {\left[\mathbf{0}_{3 \times k}\right]} \\
R \psi_{g 1}^{\prime} & R \psi_{g 2}^{\prime} & \ldots & R \psi_{g k}^{\prime}
\end{array}\right] .
$$

In order to form each modal coefficient of the generalized displacement in (7), the relation can be written as the inner product of a row vector of sums over all stations and the generalized displacement of $k$ modes, that is,

$$
\bar{q} S \sum_{x=1}^{l} l_{x}\left[\frac{\partial C_{N}}{\partial \alpha}\right] \sum_{x i=1}^{k} \psi_{i x}^{\prime} \eta_{i}=\bar{q} S\left[\sum_{x=1}^{l} l_{x}\left[\frac{\partial C_{N}}{\partial \alpha}\right]_{x} \psi_{1 x}^{\prime} \quad \sum_{x=1}^{l} l_{x}\left[\frac{\partial C_{N}}{\partial \alpha}\right]_{x} \psi_{2 x}^{\prime} \quad \ldots \quad \sum_{x=1}^{l} l_{x}\left[\frac{\partial C_{N}}{\partial \alpha}\right]_{x} \psi_{k x}^{\prime}\right] \eta
$$

with the vector generalized displacement $\eta=\left[\begin{array}{llll}\eta_{1} & \eta_{2} & \ldots & \eta_{k}\end{array}\right]$. Using the operator (15) and the matrix in (16), the row vector in (18) can be rewritten as

$$
\bar{q} S \sum_{x=1}^{l} l_{x}\left[\frac{\partial C_{N}}{\partial \alpha}\right] \sum_{x=1}^{k} \psi_{i x}^{\prime} \eta_{i}=\bar{q} S\left[\dot{\boldsymbol{\psi}}_{a}\left(\boldsymbol{l}_{\boldsymbol{x}} * \boldsymbol{d}_{\boldsymbol{x}}\right)\right]^{T} \boldsymbol{\eta},
$$

and similar results follow to accommodate the remaining terms in (7) and (8), where

$$
\begin{gathered}
-\frac{\bar{q} S}{V} \sum_{x=1}^{l} l_{x}\left[\frac{\partial C_{N}}{\partial \alpha}\right] \sum_{x}^{k} \psi_{i x} \dot{\eta}_{i}=-\frac{\bar{q} S}{V}\left[\boldsymbol{\Psi}_{\boldsymbol{a}}\left(\boldsymbol{l}_{\boldsymbol{x}} * \boldsymbol{d}_{\boldsymbol{x}}\right)\right]^{T} \dot{\boldsymbol{\eta}}, \\
\bar{q} S \sum_{x=1}^{l}\left[\frac{\partial C_{N}}{\partial \alpha}\right]_{x} \sum_{i=1}^{k} \psi_{i x}^{\prime} \eta_{i}=\bar{q} S\left[\dot{\boldsymbol{\psi}}_{a} \boldsymbol{d}_{\boldsymbol{x}}\right]^{T} \boldsymbol{\eta},
\end{gathered}
$$

and

$$
-\frac{\bar{q} S}{V} \sum_{x=1}^{l}\left[\frac{\partial C_{N}}{\partial \alpha}\right] \sum_{x=1}^{k} \psi_{i x} \dot{\eta}_{i}=-\frac{\bar{q} S}{V}\left[\boldsymbol{\Psi}_{a} \boldsymbol{d}_{\boldsymbol{x}}\right]^{T} \dot{\boldsymbol{\eta}}
$$

It follows that the flex-to-rigid coupling matrix can be compactly expressed as

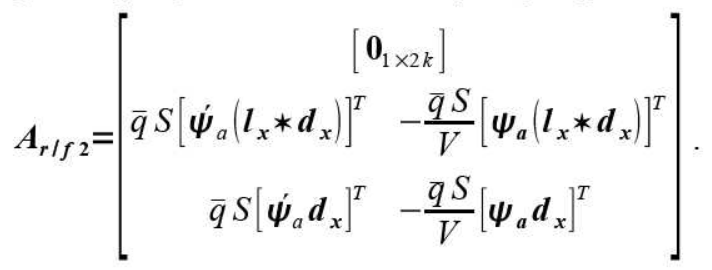

Finally, the flex to rigid coupling is the sum of the force follower effects and aeroelastic coupling, such that

$$
A_{r / f}=A_{r / f 1}+A_{r / f 2} \text {. }
$$

\section{Rigid Excitation of Flex and Intermodal Coupling}

Both rigid and flexible motion contribute to the modal forcing functions. The excitation of the $i^{\text {th }}$ of $k$ modes by forces resulting from displacements and velocities of all $k$ modes is an modal coupling effect of otherwise orthogonal modes. The generalized force on the $i^{\text {th }}$ bending mode due to rigid and flexible aerodynamic loads is represented by the right-hand side of (9).

$\dagger$ A typographical error appears in this expression in reference [6].

6

American Institute of Aeronautics and Astronautics 


\section{A. Rigid Excitation}

In the absence of distributed aerodynamics, the rigid-to-flex coupling matrix is the null matrix due to orthogonality;

$$
\boldsymbol{A}_{\boldsymbol{f} / \boldsymbol{r}}=\left[\mathbf{0}_{2 k \times 3}\right] \text {. }
$$

The first three terms on the right-hand side of (9) can be partitioned and written as coefficients of the state vector such that

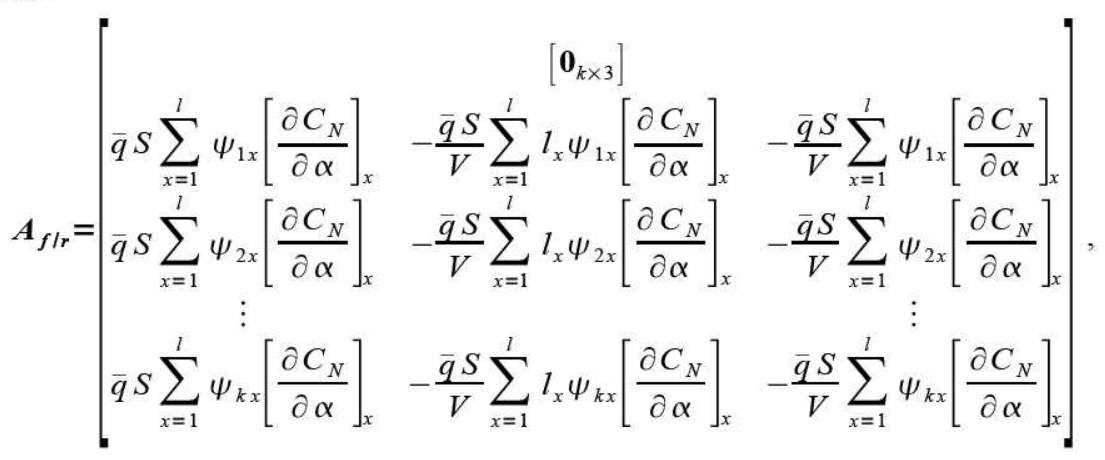

and it follows from our previous developments that (20), (21), and (22) can be used to compactly rewrite (26) as

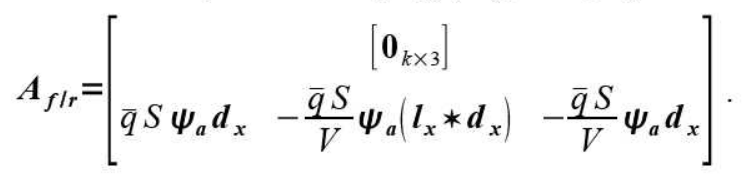

\section{B. Bending Influence Coefficients}

Finally, the remaining two terms on the right-hand side of (9) must be incorporated into the flex dynamics. Assuming normalized eigenvectors, the unforced flexibility equations have the form

$$
\left[\begin{array}{l}
\dot{\eta} \\
\ddot{\eta}
\end{array}\right]=A_{f}\left[\begin{array}{l}
\eta \\
\dot{\eta}
\end{array}\right]
$$

where

$$
\boldsymbol{A}_{f}=\left[\begin{array}{cc}
{\left[\boldsymbol{0}_{k \times k}\right]} & {\left[\boldsymbol{I}_{k \times k}\right]} \\
\boldsymbol{K}_{\boldsymbol{h}}+\boldsymbol{K}_{\boldsymbol{f}}+\boldsymbol{K}_{\boldsymbol{a}} & \boldsymbol{D}_{\boldsymbol{h}}+\boldsymbol{D}_{\boldsymbol{a}}
\end{array}\right] .
$$

The diagonal matrices $\boldsymbol{K}_{h}, \boldsymbol{D}_{\boldsymbol{h}}$ are the stiffness and damping matrices for the second-order flex dynamics; $\boldsymbol{K}_{f}$ is an augmented stiffness matrix resulting from elastic force following, ${ }^{6}$ and $\boldsymbol{K}_{a}, \boldsymbol{D}_{a}$ are the augmented stiffness and damping formed from the outer products of the mode shape and slope along the vehicle, weighted by the line load vector. Via expansion and reordering of summations, write the first vector of double sums in (9) as

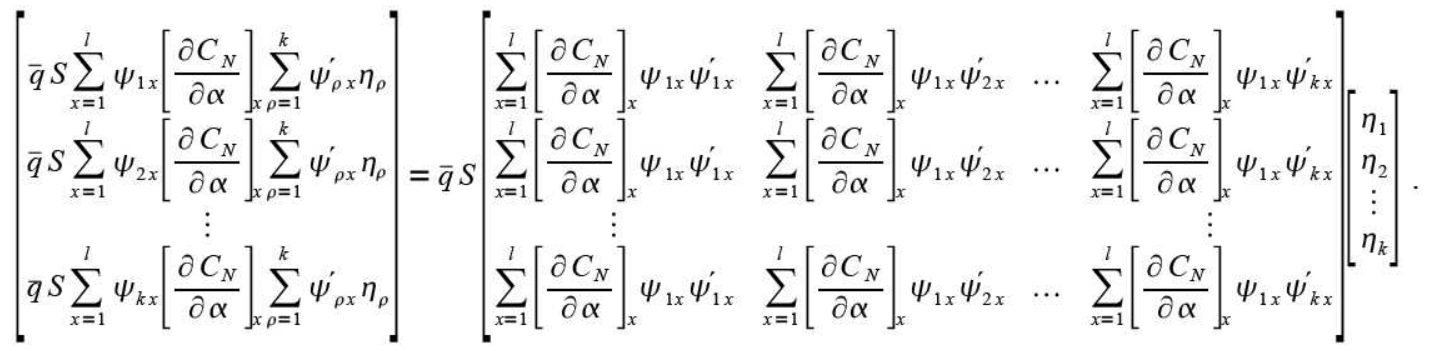

Inspection of the matrix on the right-hand side of (30) reveals that this expression can be written as the sum of $l k \times k$ matrices. 
Each $x$ of $l \quad k \times k$ matrices $\boldsymbol{\sigma}_{x}$ is formed from the dyadic product of the $x^{\text {th }}$ columns of $\boldsymbol{\psi}_{x}, \dot{\boldsymbol{\psi}}_{x}$ multiplied by the line load scalar corresponding to the $x^{\text {th }}$ station, that is,

$$
\boldsymbol{\sigma}_{x}=\left[\frac{\partial C_{N}}{\partial \alpha}\right]\left[\begin{array}{c}
\psi_{1 x} \\
\psi_{x x} \\
\vdots \\
\psi_{k x}
\end{array}\right]\left[\begin{array}{llll}
\psi_{1 x}^{\prime} & \psi_{2 x}^{\prime} & \ldots & \psi_{k x}^{\prime}
\end{array}\right],
$$

and finally, (30) can be written

$$
\left[\begin{array}{c}
\bar{q} S \sum_{x=1}^{l} \psi_{1 x}\left[\frac{\partial C_{N}}{\partial \alpha}\right] \sum_{x=1}^{k} \psi_{\rho x}^{\prime} \eta_{\rho} \\
\bar{q} S \sum_{x=1}^{l} \psi_{2 x}\left[\frac{\partial C_{N}}{\partial \alpha}\right] \sum_{x=1}^{k} \psi_{\rho x}^{\prime} \eta_{\rho} \\
\vdots \\
\bar{q} S \sum_{x=1}^{l} \psi_{k x}\left[\frac{\partial C_{N}}{\partial \alpha}\right] \sum_{x=1}^{k} \psi_{\rho x}^{\prime} \eta_{\rho}
\end{array}\right]=\bar{q}\left[\sum_{x=1}^{l} \dot{\sigma}_{x}\right] \eta=\boldsymbol{K}_{a} \boldsymbol{\eta} .
$$

The right-hand side of (32) is the product of a $k \times k$ matrix and a column vector of elastic displacements. From this, it can be generalized that the remaining term in the flexibility equation can be represented using

$$
\left[\begin{array}{c}
-\frac{q S}{V} \sum_{x=1}^{l} \psi_{1 x}\left[\frac{\partial C_{N}}{\partial \alpha}\right]_{x} \sum_{\rho=1}^{k} \psi_{\rho x} \eta_{\rho} \\
-\frac{\bar{q} S}{V} \sum_{x=1}^{l} \psi_{2 x}\left[\frac{\partial C_{N}}{\partial \alpha}\right]_{x} \sum_{\rho=1}^{k} \psi_{\rho x} \eta_{\rho} \\
\vdots \\
-\frac{\bar{q} S}{V} \sum_{x=1}^{l} \psi_{k x}\left[\frac{\partial C_{N}}{\partial \alpha}\right]_{x} \sum_{\rho=1}^{k} \psi_{\rho x} \eta_{\rho}
\end{array}\right]=-\frac{\bar{q} S}{V}\left[\sum_{x=1}^{l} \boldsymbol{\sigma}_{x}\right] \dot{\boldsymbol{\eta}}=\boldsymbol{D}_{a} \dot{\boldsymbol{\eta}},
$$

where

$$
\boldsymbol{\sigma}_{x}=\left[\frac{\partial C_{N}}{\partial \alpha}\right]_{x}\left[\begin{array}{c}
\psi_{1 x} \\
\psi_{2 x} \\
\vdots \\
\psi_{k x}
\end{array}\right]\left[\begin{array}{llll}
\psi_{1 x} & \psi_{2 x} & \ldots & \psi_{k x}
\end{array}\right]
$$

With the aeroelastic coupling matrices defined, it is noted that $\boldsymbol{K}_{a}, \boldsymbol{D}_{a}$ are linear in dynamic pressure, reference area, and velocity, and that the matrix components are a function only of the flex eigenvectors and the line load distribution. Furthermore, for homogeneous stability of the elastic response in the presence of aeroelasticity and thrust but no other generalized forces, it is sufficient that $\boldsymbol{A}_{f}$ is Hurwitz, and

$$
\mathfrak{R}\left[\lambda_{i}\right]<0 \text {. }
$$

The well-known criterion (35) predicts aeroelastic stability of free-free vibration as a function of dynamic pressure and velocity, independent of the remaining dynamics and attitude control system. It is important to note, however, that while condition (35) is necessary for homogeneous stability of the bending dynamics in the absence of non-aerodynamic forces, the result does not generalize when the remaining dynamics and/or closed-loop attitude control are incorporated. The present model allows for the evaluation of the closed-loop eigenvalues in the presence of the fully coupled system dynamics, including propellant slosh, actuator servo-dynamics, and the full autopilot. Finally, the open-loop system from gimbal input to gimbal command is a single-input, single-output (SISO) system 
that yields a frequency response. Closed-loop stability and stability margins can be readily determined using the Nyquist criterion.

\section{Gust Response}

The rigid and elastic wind response of the vehicle is driven by local lateral velocity at each station along the vehicle centerline. The rigid response to a gust input is determined from the sum of the forces and moments due to gust inputs appearing at each $x$ of $l$ stations. The existing model inputs $\boldsymbol{u}=\left[\begin{array}{ll}\beta & \ddot{\beta}\end{array}\right]$ are the nozzle position and acceleration from an actuator dynamics model. For gust penetration studies, the input can be expanded to include the unique lateral wind gust velocity at each station, resulting in an input vector

$$
\boldsymbol{u}=\left[\begin{array}{llllll}
\beta & \ddot{\beta} & w_{1} & w_{2} & \ldots & w_{l}
\end{array}\right]
$$

and input distribution matrices $\boldsymbol{B}_{r}, \boldsymbol{B}_{f}$ that are expanded to dimension $3 \times(2+l)$ and $2 k \times(2+l)$, respectively. For typical ascent flight conditions, however, the forward velocity is sufficiently high that it is reasonable to assume that the vehicle encounters the change in wind velocity at every station simultaneously. In this case, all $w_{i}=w$ as the gust velocity collapses to a single input.

If a single input is assumed, it follows from the relationships derived above that the sum of the coefficients of $w_{x}$ over all $x$ stations on the right-hand side of (7), (8) can be represented using the same rigid lateral velocity entries in (13). The rigid-body input coupling matrix is expanded such that

$$
\boldsymbol{B}_{r}=\left[\begin{array}{c|c|c} 
& & 0 \\
0 & 0 & \frac{\bar{q} S k_{r}}{V} \\
-l_{g} R & -l_{g} l_{n} m_{n}-I_{n} & \bar{q} S k_{t} \\
R & l_{n} m_{n} & \frac{V}{V}
\end{array}\right] .
$$

Likewise, the flex input distribution matrix that accounts for the right-hand side of (9) is formed from the product of the mode shape matrix (16) and the load distribution (6), using the same coefficients as those for lateral velocity shown on the rightmost column of (26). The augmented flex input distribution matrix therefore becomes

$$
\boldsymbol{B}_{f}=\left[\begin{array}{c|c|c} 
& {\left[\mathbf{0}_{k \times 3}\right]} \\
R \psi_{g 1} & l_{n} m_{n} \psi_{g 1}-I_{n} \psi_{g 1}^{\prime} & \\
R \psi_{g 2} & l_{n} m_{n} \psi_{g 2}-I_{n} \psi_{g 2}^{\prime} & \bar{q} S \\
\ldots & \cdots & \\
R \psi_{g k} & l_{n} m_{n} \psi_{g k}-I_{n} \psi_{g k}^{\prime} &
\end{array}\right] .
$$

\section{Simulation}

The present formulation has been incorporated into a general missile autopilot stability analysis and design tool, FRACTAL. ${ }^{6}$ FRACTAL allows the trajectory to be simulated at a particular flight condition under the assumptions of fixed (LTI) or time-varying (LTV) parameters so as to assess the impact of variations in a given vehicle configuration and autopilot design on the relative stability of the closed-loop system. The perturbation dynamics are inherently linear and the use of classical stability margins is appropriate.

As is often the case with large flexible boosters, phase stabilization of the first bending mode is a requirement to ensure high robustness to bending mode uncertainty; as such, the system is conditionally stable in phase. Since large boosters tend to be aerodynamically unstable unless augmented with external aerodynamic stabilization devices, the system is conditionally stable in gain. Various assessments of coupled transient response and stability have been performed, a subset of which is discussed below. 


\section{A. Root loci of the aeroelastic dynamics}

Use of the flexibility equations (28) alone provides the open-loop eigenvalues of the elastic response in the presence of a thrust follower force and aeroelastic forces at various thrust levels and dynamic pressures and can be readily obtained by varying the parameters in (29). A representative configuration of the Ares I vehicle was incorporated into the present model and various root loci were computed at dynamic pressures ranging from zero to the maximum dispersed dynamic pressure for a given flight condition. ${ }^{8}$ An example of the root locus of elastic eigenvalues is shown in Figure 3.

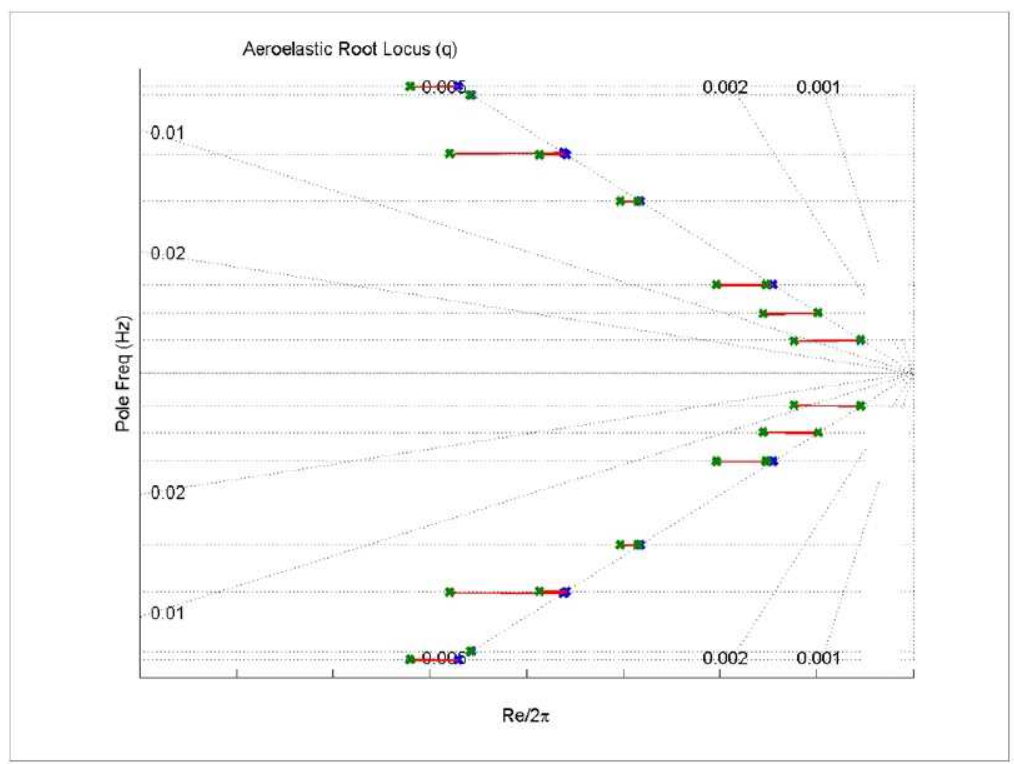

Figure 3: Aeroelastic root locus near maximum dynamic pressure

It is clear from the figure that the general trend is an increase in aerodynamic damping from the assumed structural damping value of $\zeta_{b i}=0.005$ to as much as $\zeta_{b i}=0.01$ for the lower frequency modes. Very little frequency shift (due to rotational coupling and force follower effects) is observed for this vehicle configuration; the dominant effect is due to the translational degrees of freedom.

Conclusions regarding modal damping must consider the nature of the planar, rigid load approximation and the limited fidelity in the higher frequency modes. An increase in modal damping in the linear analysis is reassuring, but is not definitive evidence that modal damping is increasing. It would be unwise, therefore, to rely on this modal damping for control design.

\section{B. Stability margin assessment}

An investigation of stability margins using the integrated linear formulation allows one to determine the relative impact of aeroelasticity on the vehicle stability margins with all other dynamics (propellant slosh, nozzle degrees of freedom) included in the analysis. An LTI Monte Carlo analysis is conducted at each operating point of interest and various stability margins are extracted and verified using an automated process. The most significant impact to the open-loop stability with the autopilot included can be predicted from the preceding eigenvalue analysis: attenuation of flexibility due to increased structural damping has a marked impact on the elastic response.

A comparison of the nominal frequency responses is presented in a Nichols plot in Figure 1. The inclusion of the distributed line loads has the anticipated effect shown from the eigenvalue analysis. The amplitude of the first bending mode is decreased due to the increase in aerodynamic damping, resulting in an increase in high-frequency phase margin for the phase-stabilized first bending mode. Interestingly, slosh mode response is also affected due to coupling through bending. 


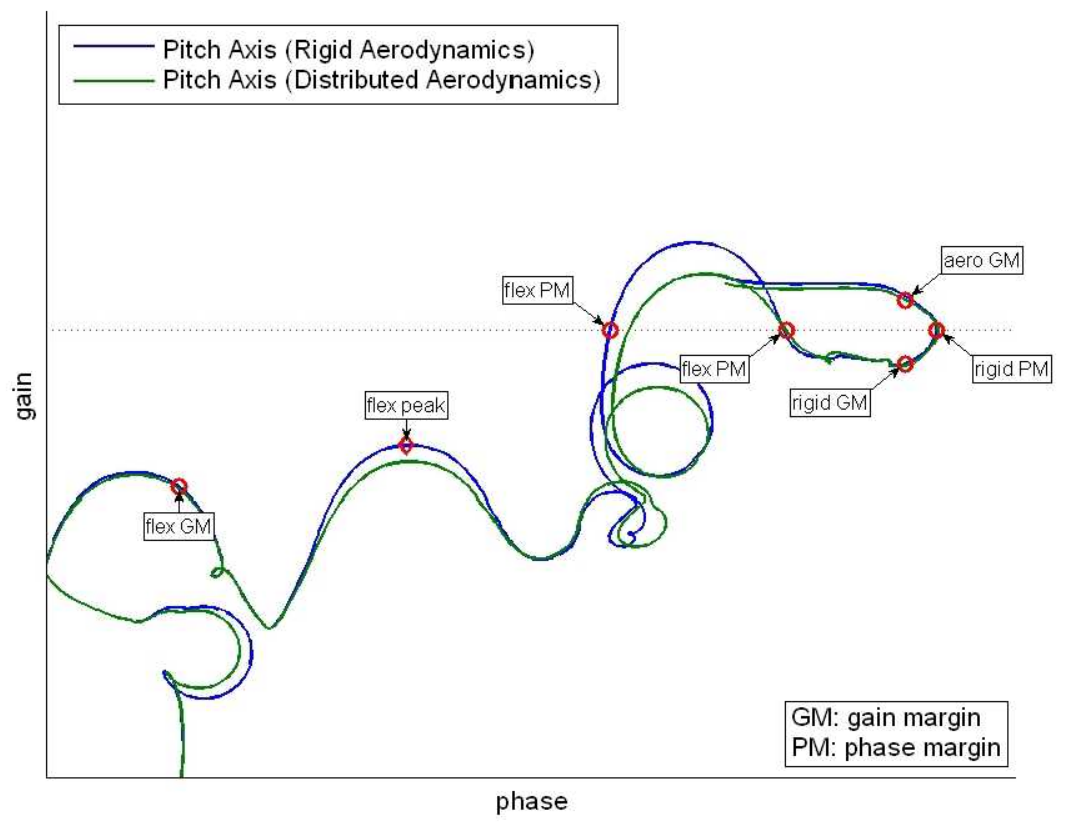

Figure 4: Nichols response of coupled model

For Monte Carlo evaluation of stability margins, several tens of thousands of cases are analyzed with various parameter dispersions sourced from differing vehicle trajectories. An example of a Monte Carlo analysis performed at various flight conditions is shown below in Figures 5 and 6 . The effects of rigid line loads on the coupled open-loop autopilot stability are evident as a general trend of decreased aerodynamic gain margin near maximum dynamic pressure. Of course, this effect is well within the vehicle and autopilot design capabilities ${ }^{\S}$, but it illustrates the more aggressive rigid body low-frequency aerodynamic response encountered via the use of rigid distributed loads data in the fully coupled model.

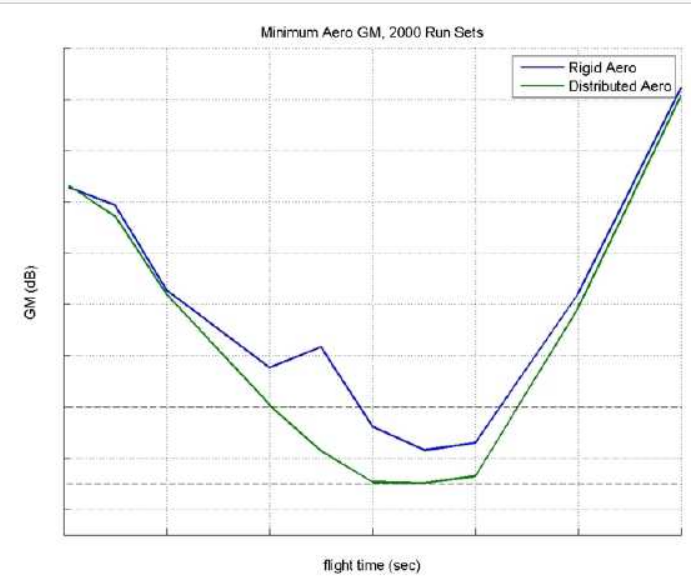

Figure 5: Monte Carlo minimum aerodynamic gain margin

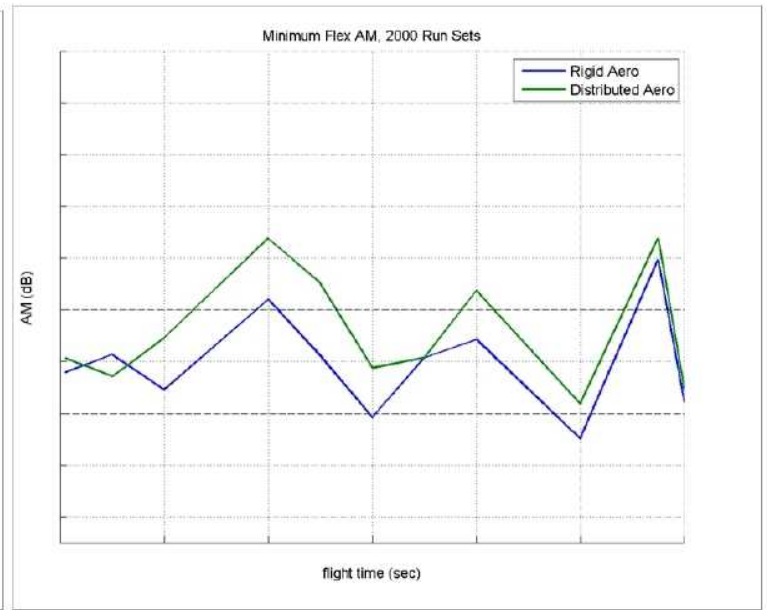

Figure 6: Monte Carlo peak elastic repsonse

$\S$ While the aerodynamic gain margin tends to decrease with the inclusion of rigid line loads, the rigid body phase margin increases due to aerodynamic pitch damping, potentially allowing for improved performance via additional autopilot tuning. 
The effects of aerodynamic damping on the higher frequency modes are presented in Figure 6. As was seen in the nominal stability analysis, the flex attenuation or minimum flex amplitude margin (typically driven by the second lateral bending mode) increases due to the aerodynamic damping effect. The trend throughout flight is an overall decrease in the open-loop elastic response sensed by the rate gyros of the attitude control system. Such a decrease is beneficial as decreased bending filter attenuation requirements potentially allow for improved controller bandwidth and attitude tracking performance, given sufficient confidence in the aerodynamics model.

\section{Coupled transient response analysis}

A visualization tool has been constructed with a dynamics engine based upon the FRACTAL model architecture. The visualization system is intended to allow for rapid evaluation of autopilot response to gust loads and disturbances at various frozen-time flight conditions through animation. For example, the onset of a steady-state wind or wind profile can be simulated to evaluate the ability of the in-flight load relief system to turn the vehicle into the wind. Lateral bending, slosh, and nozzle deflections are depicted in real time, and trajectoryrelative position and velocity errors are computed. An example of the output of the visualization tool is shown below in Figure 7. The response to a steady-state wind disturbance has been simulated to evaluate the bending dynamics and trajectory error.

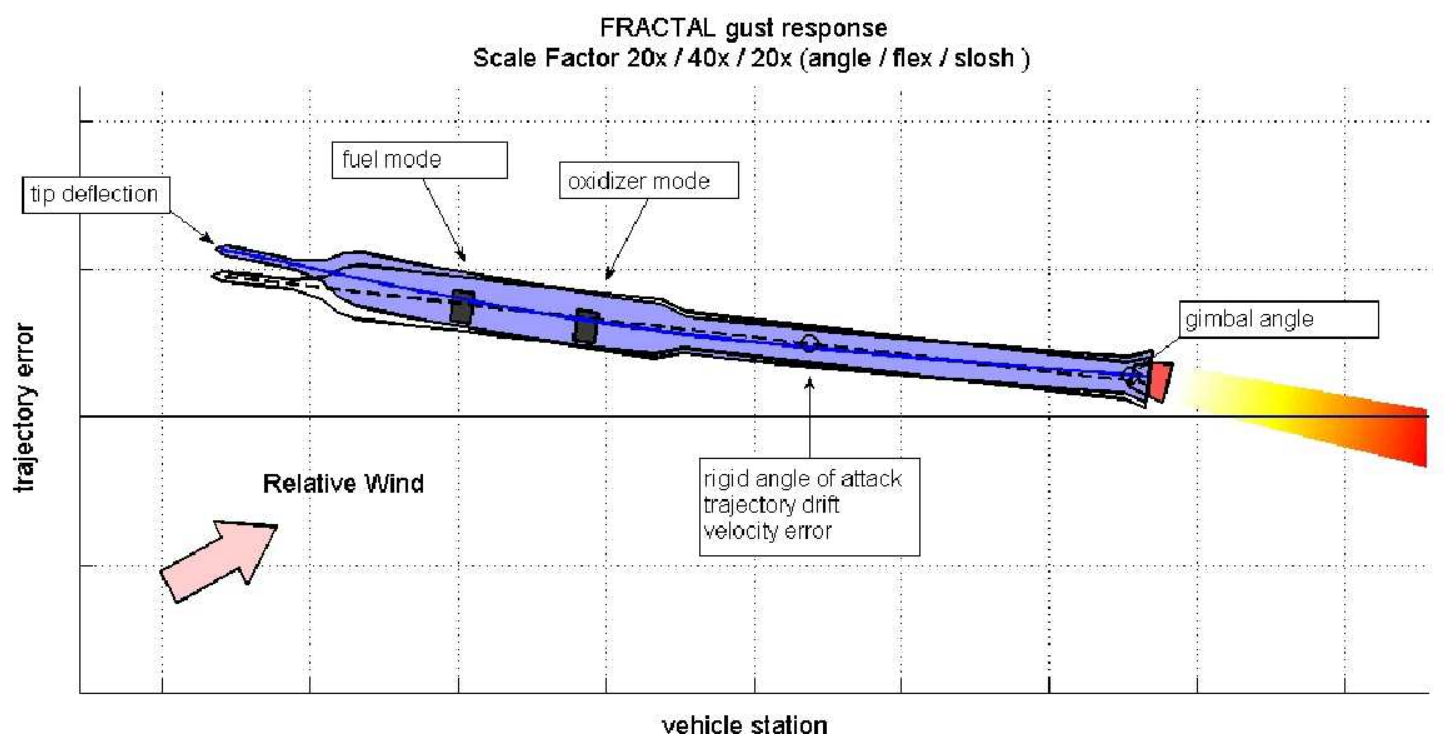

Figure 7: Transient response simulation

\section{Discussion}

The present work has illustrated a technique for the incorporation of a planar aeroelastic model into the equations of motion for stability analysis of a traditional launch vehicle. The approach is based on the classical perturbation methods for stability assessment. While the model is linear and is a first-order approximation to the true dynamics encountered in a non-steady-state load condition, the general trends can be expected to hold so long as the vehicle is indeed fairly rotationally symmetric and the assumption of uncoupled motion in a single plane is realistic. While the present model cannot predict the complex interacting physics that can be simulated in coupled computational fluid dynamics (CFD)/finite element model (FEM) codes, it benefits from being compact and extremely efficient to implement in a matrix-based programming language. Rapid execution allows for the formulation to be used within optimization routines and for rapid Monte Carlo stability analysis. Further work will compare the present results with evolving higher-fidelity data to assess the accuracy of the technique when compared with large-scale nonlinear multiphysics simulations of the vehicle dynamics. 


\section{Acknowledgments}

This work was prepared by bd Systems, an SAIC company, for the NASA Marshall Space Flight Center under contract to MSFC (NNM06AA01Z). The author extends thanks to Bandu Pamadi and Bob Hall of NASA Langley Research Center for their insight and assistance in providing data for the analysis.

\section{References}

${ }^{1}$ Lukens, D., Schmitt, A., and Broucek, G., "Approximate Transfer Functions for Flexible Booster and Autopilot Analysis," WADD-TR-61-93, 1961.

${ }^{2}$ Rheinfurth, M. H., “Control-Feedback Stability Analysis,” ABMA DA-TR-2-60, 1960.

${ }^{3}$ Frosch, J. and Vallely, D., "Saturn AS-501/S-IC Flight Control System Design," J. Spacecraft, Vol. 4, No. 8, 1967, p. 1003-1009.

${ }^{4}$ Garner, D., "Control Theory Handbook," NASA TM-X-53036, 1964.

${ }^{5}$ Orr, J., "FRACTAL Linear Model Dynamics," NASA MSFC/EV41 (TCD20090535A), April 29, 2009.

${ }^{6}$ Orr, J., Johnson, M., Wetherbee, J., and McDuffie, J., "State Space Implementation of Linear Perturbation Dynamics Equations for Flexible Launch Vehicles," AIAA 2009-5962, August 2009.

${ }^{7}$ Alley, V.L. and Harper-Gerringer, A., "An Analysis of Aeroelastic Divergence in Unguided Launch Vehicles," NASA TN D-3281, March 1966.

8“Flutter, Buzz, and Divergence." NASA Space Vehicle Design Criteria, SP-8003, 1964.

${ }^{9}$ O'Keefe, D.A., "General Static Aeroelastic Analysis for a Body of Revolution," NASA TN D-3281, March 1966. 


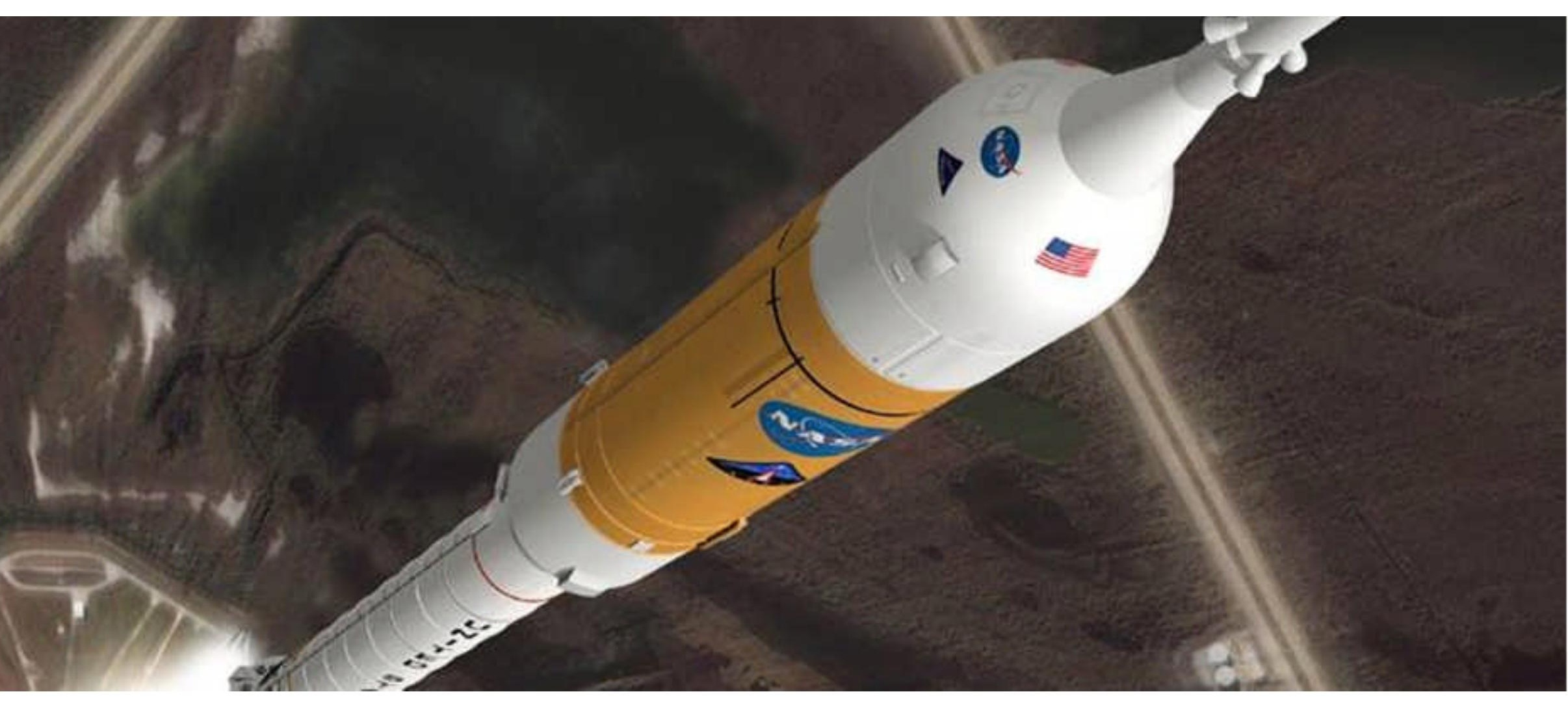

\section{A Coupled Aeroelastic Model for Launch Vehicle Stability Analysis}




\section{Overview and Objectives}

- Motivations for a coupled aeroelastic model

- Challenges in launch vehicle control

- Existing approaches in analysis of flexible rocket dynamics

- Assumptions and limitations

- Model derivation and implementation

- Example results 


\section{Motivations}

- From a vehicle integration perspective, aeroelasticity, loads, and closed-loop autopilot stability are considered decoupled problems

- Aeroelastic stability is verified via extensive analysis assuming free-free structural modes; loads are verified using resultant modal damping from active stabilization (controller in the loop) and aeroelastic damping (positive or negative)

- Control stability is verified using rigid-body aerodynamics with a coupled rigid-flexslosh-actuator model

- Recent work has focused on revisiting and improving heritage launch vehicle dynamics models using modern linear algebra tools

- Numerical recipes for constructing high order linear models have been developed

- These models are fully coupled within the linearity assumptions (well justified for traditional launch vehicle configurations)

- Present work incorporates rigid line loads into the fully-coupled model

- Allow evaluation of autopilot stability in the presence of an aeroelastic system

- Enhance confidence of aeroelastic stability in the presence of closed loop control 


\section{Challenges in Launch Vehicle Control}

- Complex dynamic models

- Nonlinear, coupled, flexible multi-body system with variable mass

- Flexible body models can be very high order

- Limited controller architecture

- Linear controllers prefaced with bending filters are preferred based on flight heritage

- Highly flexible

- Sensed angles and rates are corrupted by vibration

- Structural oscillations must be mitigated to minimize bending loads, aerodynamics, and propellant motion

- Conditionally stable (aerodynamics and phase-stable bending mode)

- Large uncertainties in key parameters

- Significant dynamic coupling (flex, slosh, aerodynamics, nozzle dynamics) 


\section{Perturbation Dynamics History}

- Small perturbations have classically been used in assessing short-period stability of aircraft about a trim condition

- Launch vehicle models were derived from similar assumptions and matured through the 1950s-1960s

- Perturbation models are well suited to launch vehicles due to the small deflections from the trajectory encountered in the ascent environment

- For example, gravity turn trajectories with small angles of attack

- Classical models were of reduced order, and derived in transfer function or block form for implementation on an analog computer or for computing frequency response

- Control design often performed using root-locus methods, resulting in a PD-type controller prefaced by bending filters that removed elastic response from the autopilot attitude and rate channels 


\section{Modeling Assumptions}

- General assumptions

- Vehicle is tetragonally symmetric and exhibits negligible pitch/yaw coupling

- Reference trajectory is a gravity turn where $g$ can be ignored:

$$
\dot{x}_{C}=\frac{g \sin x_{C}}{V}
$$

- Vehicle undergoes small perturbations from the reference trajectory, and elasticity can be represented in the linear range

- Propellant slosh can be modeled via a spring-mass-damper mechanical analogue

- Aerodynamics are well-behaved and can be linearized about zero angle of attack

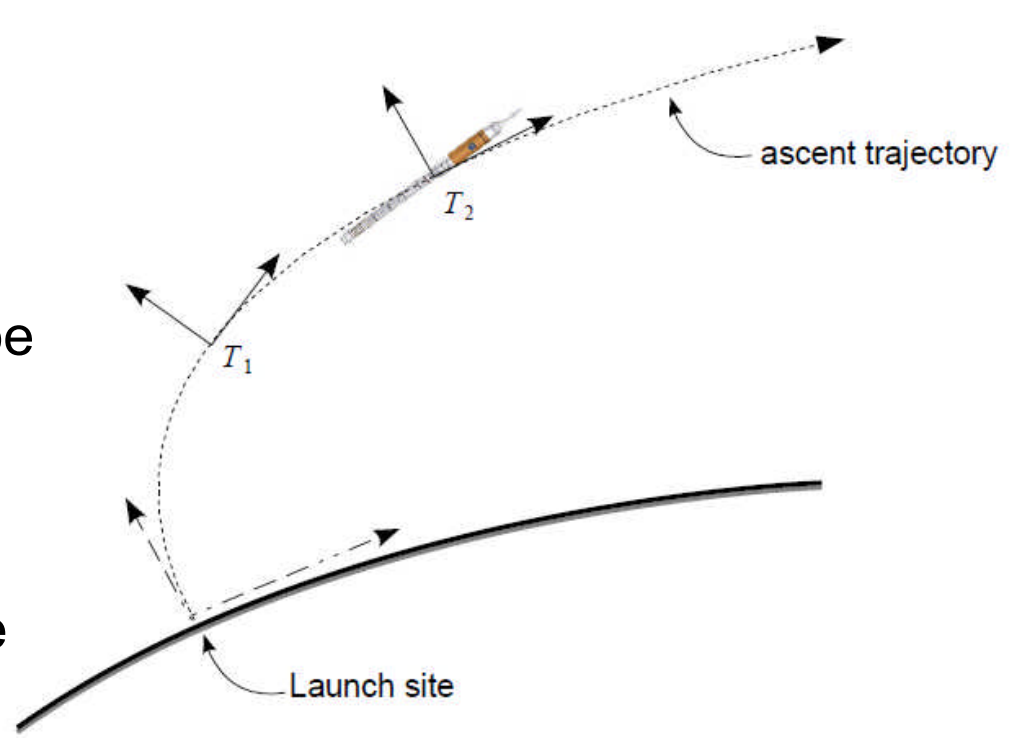

- Quasi-inertial frame is a trajectory frame that translates axially with the vehicle (2-DoF) 


\section{Modeling Assumptions (II)}

- Vehicle is represented by a quasi-rigid base body on which orthogonal elastic modes are superimposed

- Linear elasticity represented via a set of assumed modes (eigenvectors) and frequencies (eigenvalues)

- Other dynamic modes (propellant, actuator, and nozzle dynamics) are coupled to the base body

- Complete system dynamics can be compactly derived from the Lagrangian

- The present formulation assumes 2 rigid DoF (rotation, translation), $n$ slosh DoF, and $k$ flex DoF.

- The system dynamics are implemented in first order descriptor form as

$$
E \dot{x}=A x+B u, \quad y=C x+D u
$$

- Controller, actuator, etc. are added via block diagonalization

- A vehicle with a single engine or cluster of engines can be analyzed as SISO at the gimbal break 


\section{Modeling Assumptions (III)}

- The aeroelastic model uses a quasi-rigid line load distribution

- Line loads derived from wind tunnel and CFD tests about zero angle of attack

- Does not account for nonstationary effects; e.g., the force distribution derivative is not a function of the flexible motion

- The continuous distribution is reduced to a discrete distribution integrated over intervals corresponding to FEM gridpoints attached to the load structure

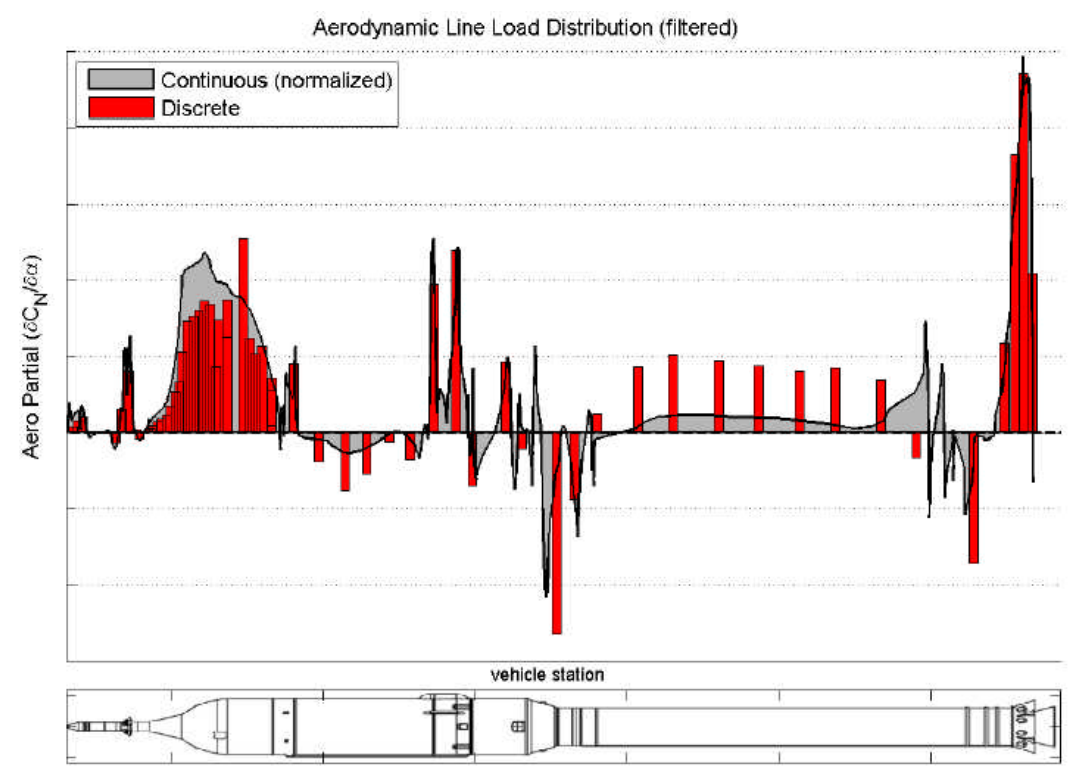




\section{Reference Frame Geometry}

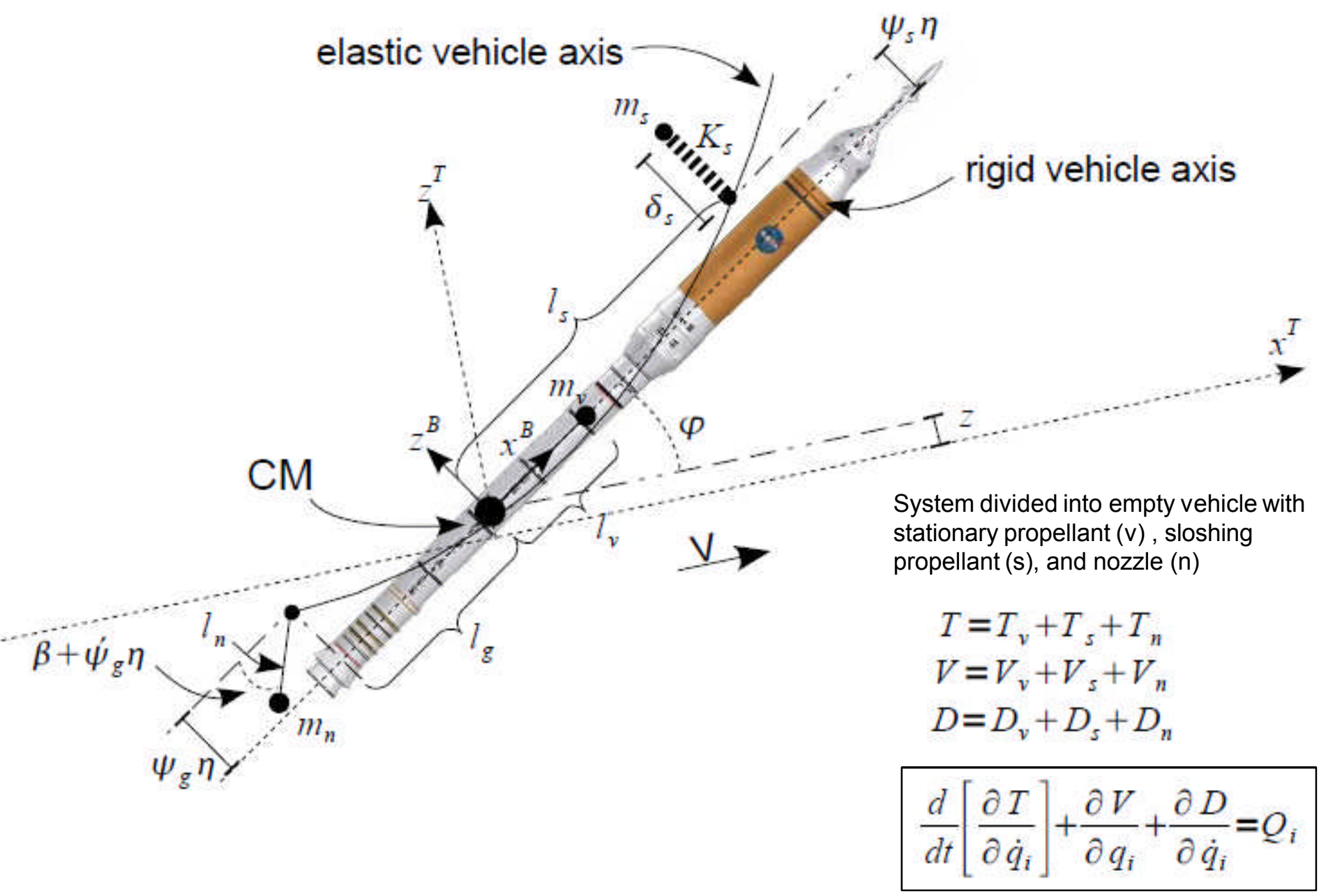




\section{Linear Dynamics Model}

- Derivation of the coupled dynamics yields a block linear model of the form

$$
\begin{aligned}
& \left.\left[\begin{array}{c}
\boldsymbol{E}_{r} \\
\boldsymbol{E}_{f} \\
\boldsymbol{E}_{s}
\end{array}\right]\left[\begin{array}{c}
\dot{\boldsymbol{x}}_{r} \\
\dot{\boldsymbol{x}}_{f} \\
\dot{\boldsymbol{x}}_{s}
\end{array}\right]=\left[\begin{array}{ccc}
\boldsymbol{A}_{\boldsymbol{r}} & \boldsymbol{A}_{r / f} & \boldsymbol{A}_{r / s} \\
\boldsymbol{A}_{f / r} & \boldsymbol{A}_{f} & \boldsymbol{A}_{f / s} / s \\
\boldsymbol{A}_{s / r} & \boldsymbol{A}_{s / f} & \boldsymbol{A}_{s}
\end{array}\right]\left[\begin{array}{c}
\boldsymbol{x}_{r} \\
\boldsymbol{x}_{f} \\
\boldsymbol{x}_{s}
\end{array}\right]+\left[\begin{array}{l}
\boldsymbol{B}_{r} \\
\boldsymbol{B}_{f} \\
\boldsymbol{B}_{s}
\end{array}\right] \mid u_{1} u_{2} \ldots u_{n}\right], \quad \boldsymbol{y}=\left[\begin{array}{l}
\boldsymbol{C}_{m=1 \ldots p} \\
\boldsymbol{C}_{m=1 \ldots q} \\
\boldsymbol{C}_{m=1 \ldots r}
\end{array}\right]\left[\begin{array}{c}
\boldsymbol{x}_{r} \\
\boldsymbol{x}_{f} \\
\boldsymbol{x}_{s}
\end{array}\right]+\left[\begin{array}{l}
\boldsymbol{D}_{m=1 \ldots p} \\
\boldsymbol{D}_{m=1 \ldots q} \\
\boldsymbol{D}_{m=1 \ldots r}
\end{array}\right] u \\
& \boldsymbol{x}=\left[\begin{array}{lllllllllllllllllll}
\varphi & \dot{\varphi} & z & \eta_{1} & \eta_{2} & \ldots & \eta_{k} & \dot{\eta}_{1} & \dot{\eta}_{2} & \ldots & \dot{\eta}_{k} & \delta_{s 1} & \delta_{s 2} & \ldots & \delta_{s n} & \dot{\delta}_{s 1} & \dot{\delta}_{s 2} & \ldots & \dot{\delta}_{s n}
\end{array}\right]^{T}
\end{aligned}
$$

- For a rigid rocket, the angle of attack is invariant along the centerline and is given by

$$
\alpha=\varphi-\frac{\dot{z}}{V}+\frac{w}{V}
$$

with the rigid dynamics

$$
\boldsymbol{A}_{r}=\left[\begin{array}{ccc}
0 & 1 & 0 \\
-\frac{\partial C_{N}}{\partial \alpha} \bar{q} S l_{a} & 0 & \frac{\partial C_{N}}{\partial \alpha} \frac{\bar{q} S}{V} l_{a} \\
M \bar{g}+\frac{\partial C_{N}}{\partial \alpha} \bar{q} S & 0 & -\frac{\partial C_{N}}{\partial \alpha} \frac{\bar{q} S}{V}
\end{array}\right]
$$




\section{Local Angle of Attack}

- For the flexible vehicle, the local angle of attack along the vehicle centerline is a function of rotation, lateral velocity, and local gust velocity

$$
F_{a_{x} x}=\bar{q} A\left[\frac{\partial C_{N}}{\partial \alpha}\right]_{x} \alpha_{x}
$$

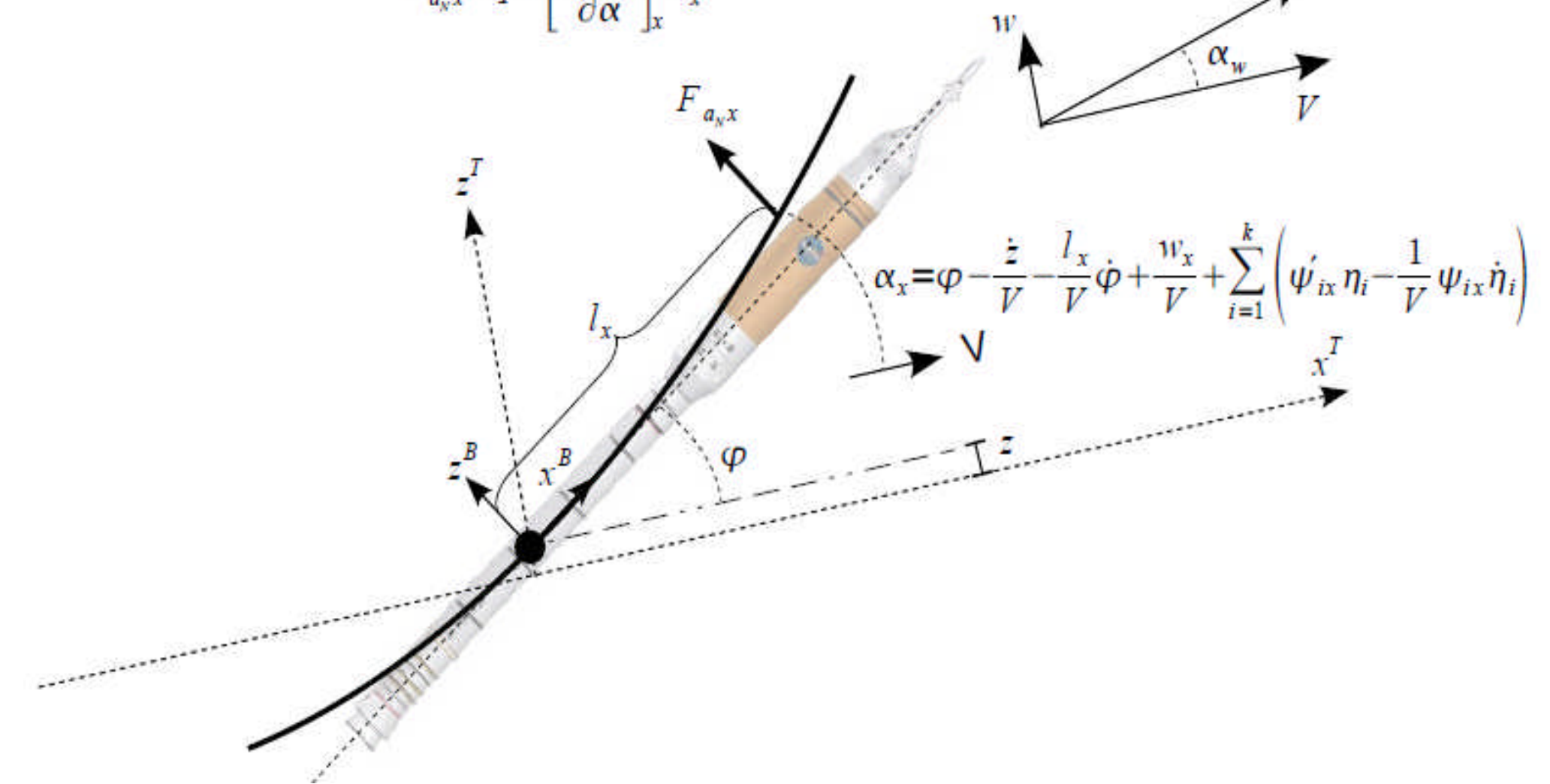




\section{Model Dynamics with}

\section{Distributed Angle of Attack}

- Model dynamics for rigid rotation, translation, and bending without other generalized forces and coupling (thrust, slosh, etc.)

$$
\begin{gathered}
I \ddot{\varphi}=\bar{q} S \sum_{x=1}^{l} l_{x}\left[\frac{\partial C_{N}}{\partial \alpha}\right]_{x}\left(\varphi-\frac{\dot{z}}{V}-\frac{l_{x}}{V} \dot{\varphi}+\frac{w_{x}}{V}+\sum_{i=1}^{k} \psi_{i x}^{\prime} \eta_{i}-\frac{1}{V} \sum_{i=1}^{k} \psi_{i x} \dot{\eta}_{i}\right) \\
M \ddot{z}-M \bar{g} \varphi=\bar{q} S \sum_{x=1}^{l}\left[\frac{\partial C_{N}}{\partial \alpha}\right]_{x}\left(\varphi-\frac{\dot{z}}{V}-\frac{l_{x}}{V} \dot{\varphi}+\frac{w_{x}}{V}+\sum_{i=1}^{k} \psi_{i x}^{\prime} \eta_{i}-\frac{1}{V} \sum_{i=1}^{k} \psi_{i x} \dot{\eta}_{i}\right) . \\
\mu_{i}\left(\ddot{\eta}_{i}+2 \zeta_{b i} \omega_{b i} \dot{\eta}+\omega_{b i}^{2} \eta_{i}\right)=\bar{q} S \sum_{x=1}^{l} \psi_{i x}\left[\frac{\partial C_{N}}{\partial \alpha}\right]_{x}\left(\varphi-\frac{\dot{z}}{V}-\frac{l_{x}}{V} \dot{\varphi}+\frac{w_{x}}{V}+\sum_{\rho=1}^{k} \psi_{\rho x}^{\prime} \eta_{\rho}-\frac{1}{V} \sum_{\rho=1}^{k} \psi_{\rho x} \dot{\eta}_{\rho}\right)
\end{gathered}
$$

- Linear model is modified with the following aeroelastic effects:

- Rigid body aerodynamic damping

- Rigid-flex coupling through aerodynamics

- Flex-flex coupling through aerodynamics (deorthogonalization)

- Wind gust inputs 


\section{Rigid Body Aerodynamic Damping}

- Rigid damping coefficients reduce to angular rate and translational terms, such that

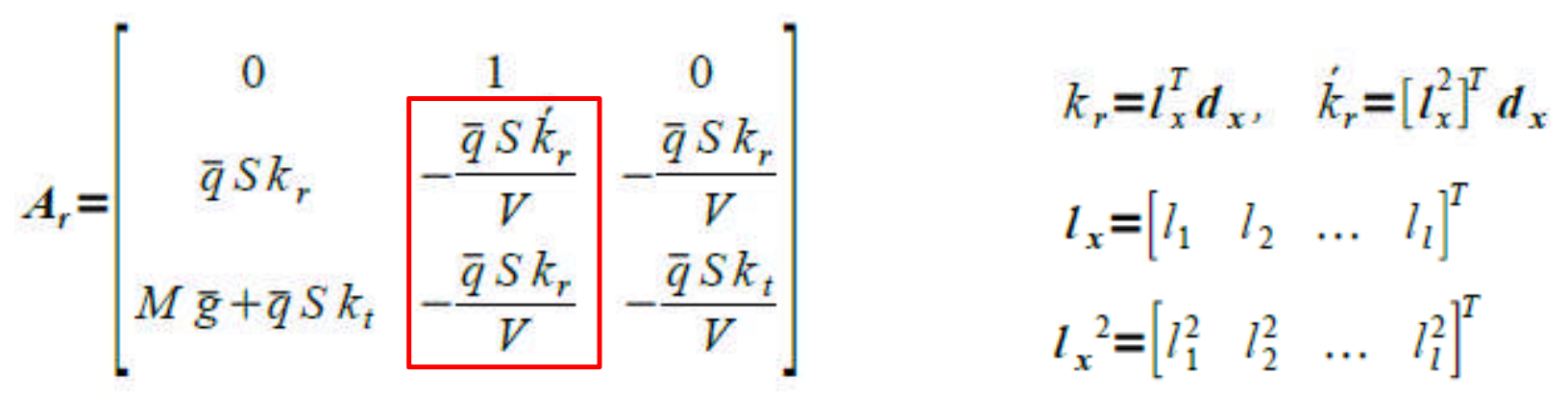

- If the rigid line loads integrate to the 6-DoF force and moment linearization, the remaining coefficients are identical 


\section{Aeroelastic-Rigid Body Coupling}

- Let $\boldsymbol{a} * \boldsymbol{b}=\left[\begin{array}{llll}a_{1} b_{1} & a_{2} b_{2} & \ldots & a_{n} b_{n}\end{array}\right]$

and

$$
\boldsymbol{\Psi}_{a}=\left[\begin{array}{cccc}
\psi_{11} & \psi_{12} & \ldots & \psi_{1 l} \\
\psi_{21} & \psi_{22} & \ldots & \psi_{2 l} \\
\vdots & & & \vdots \\
\psi_{k 1} & \psi_{k 2} & \ldots & \psi_{k l}
\end{array}\right], \quad \dot{\psi}_{a}=\left[\begin{array}{cccc}
\dot{\psi}_{11}^{\prime} & \psi_{12}^{\prime} & \ldots & \dot{\psi}_{11} \\
\dot{\psi}_{21}^{\prime} & \dot{\psi}_{22}^{\prime} & \ldots & \dot{\psi}_{2 l} \\
\vdots & & & \vdots \\
\psi_{k 1}^{\prime} & \dot{\psi}_{k 2}^{\prime} & \ldots & \dot{\psi}_{k l}^{\prime}
\end{array}\right]
$$

- Rigid coupling from flexibility via aero reduces to

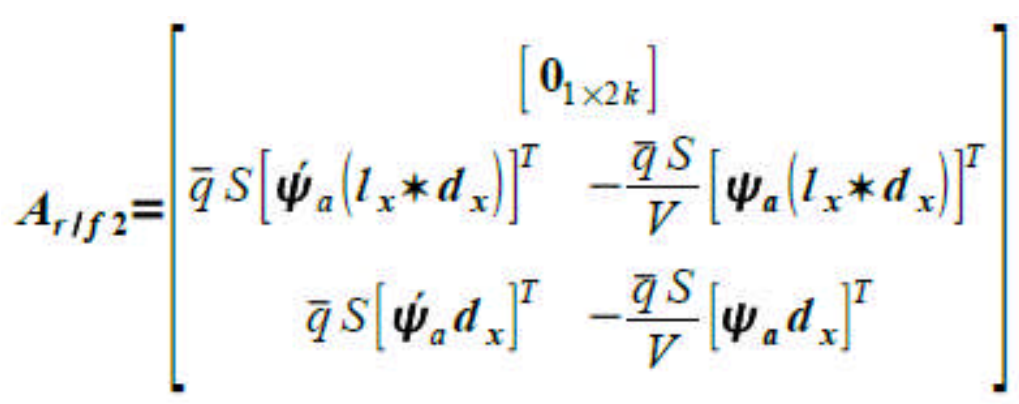

- And conversely,

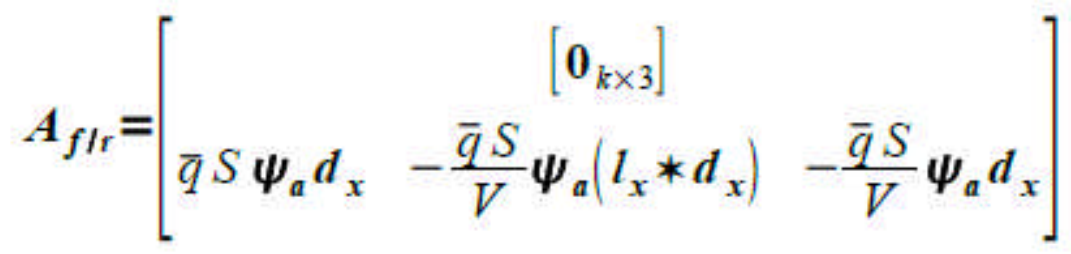




\section{Flexibility}

- Define weighted dyadics of the eigenvectors, such that

$$
\boldsymbol{\sigma}_{x}=\left[\frac{\partial C_{N}}{\partial \alpha}\right]_{x}\left[\begin{array}{c}
\psi_{1 x} \\
\psi_{2 x} \\
\vdots \\
\psi_{k x}
\end{array}\right]\left[\begin{array}{llll}
\psi_{1 x} & \psi_{2 x} & \ldots & \psi_{k x}
\end{array}\right] \quad \boldsymbol{\sigma}_{x}=\left[\frac{\partial C_{N}}{\partial \alpha}\left[\begin{array}{c}
\psi_{1 x} \\
\psi_{2 x} \\
\vdots \\
\psi_{k x}
\end{array}\right]\left[\begin{array}{llll}
\psi_{1 x}^{\prime} & \psi_{2 x}^{\prime} & \ldots & \psi_{k x}^{\prime}
\end{array}\right]\right.
$$

- Flexibility cross coupling collapses to delta stiffness and damping, and

$$
\bar{q} S\left[\sum_{x=1}^{l} \dot{\boldsymbol{\sigma}}_{x}\right] \boldsymbol{\eta}=\boldsymbol{K}_{a} \boldsymbol{\eta} \quad-\frac{\bar{q} S}{V}\left[\sum_{x=1}^{l} \boldsymbol{\sigma}_{x}\right] \dot{\boldsymbol{\eta}}=\boldsymbol{D}_{a} \dot{\boldsymbol{\eta}}
$$

- Finally, the flex response decoupled from rigid-body, actuator, and slosh dynamics is given by

$$
\left[\begin{array}{l}
\dot{\boldsymbol{\eta}} \\
\ddot{\boldsymbol{\eta}}
\end{array}\right]=\boldsymbol{A}_{f}\left[\begin{array}{l}
\boldsymbol{\eta} \\
\dot{\boldsymbol{\eta}}
\end{array}\right] \quad \boldsymbol{A}_{f}=\left[\begin{array}{cc}
{\left[\boldsymbol{0}_{k \times k}\right]} & {\left[\boldsymbol{I}_{k \times k}\right]} \\
\boldsymbol{K}_{\boldsymbol{h}}+\boldsymbol{K}_{f}+\boldsymbol{K}_{a} & \boldsymbol{D}_{\boldsymbol{h}}+\boldsymbol{D}_{a}
\end{array}\right]
$$




\section{Gust Response}

- Gust velocity is assumed small with respect to forward velocity

- A special case of the distributed gust

- Assume wind velocities are equal along the centerline

- The same rigid-body and flex coupling terms can be used for wind gust inputs

$$
\left.\boldsymbol{B}_{r}=\left[\begin{array}{cc} 
& 0 \\
\ldots & \frac{\bar{q} S k_{r}}{V} \\
& \frac{\bar{q} S k_{t}}{V}
\end{array}\right] \quad \boldsymbol{B}_{f}=\left[\begin{array}{ll} 
\\
\end{array}\right] \quad \frac{\bar{q} S}{V} \boldsymbol{\Psi}_{a} \boldsymbol{d}_{\boldsymbol{x}}\right]
$$




\section{Applications}

- Ares I

- Two stage crew launch vehicle

- Shuttle-derived five-segment solid propellant booster

- Saturn-derived upper stage powered by LOX/LH2 J-2X

- 56,000 lbm payload capacity

- $\quad>3 \mathrm{M}$ lbf thrust at liftoff

- FS and US two-axis TVC control supplemented by roll control thrusters

- $\quad$ 10 minute ascent to LEO

- Dynamically similar to classic LV designs

- Ares I-X

- First stage test vehicle with simulated upper stage

- $\quad$ 120 second flight to Mach 4+

- Flew successfully in October 2009

- Other derivatives and study vehicles
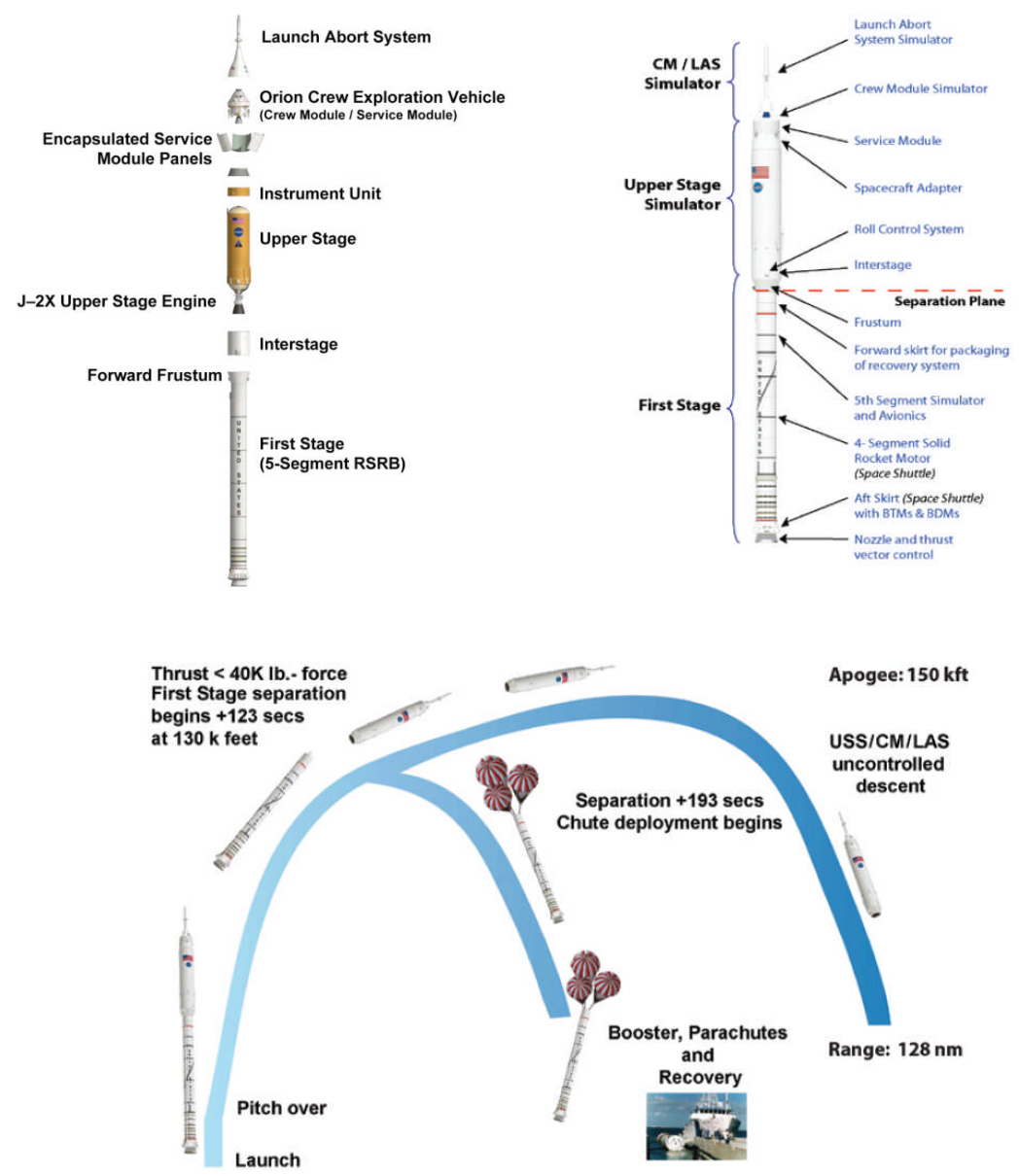


\section{Summary of Results}

- Augmented equations of motion implemented in FRACTAL (Frequency Response Analysis and Comparison Tool Assuming Linearity) $m$-code tool

- Optional aeroelasticity included in standard production runs

- First stage control design (gains/filters, sensor location studies, architecture trades)

- Monte Carlo stability analysis

- Parameter optimization (slosh damping)

- In-flight load relief performance and coupled response simulation

- Sensitivity analysis

- General aeroelasticity trends as expected for a generic launch vehicle model

- High margin for flutter and divergence

- Increase in rigid pitch (yaw) damping

- Aeroelastic damping of pitch (yaw) lateral bending modes

- Improved control stability

- Increased vehicle transient bending deflections with distributed loads 


\section{Aeroelastic Loci}

- Typical variation $1.32 x \max q$ (NASA SP-8003)
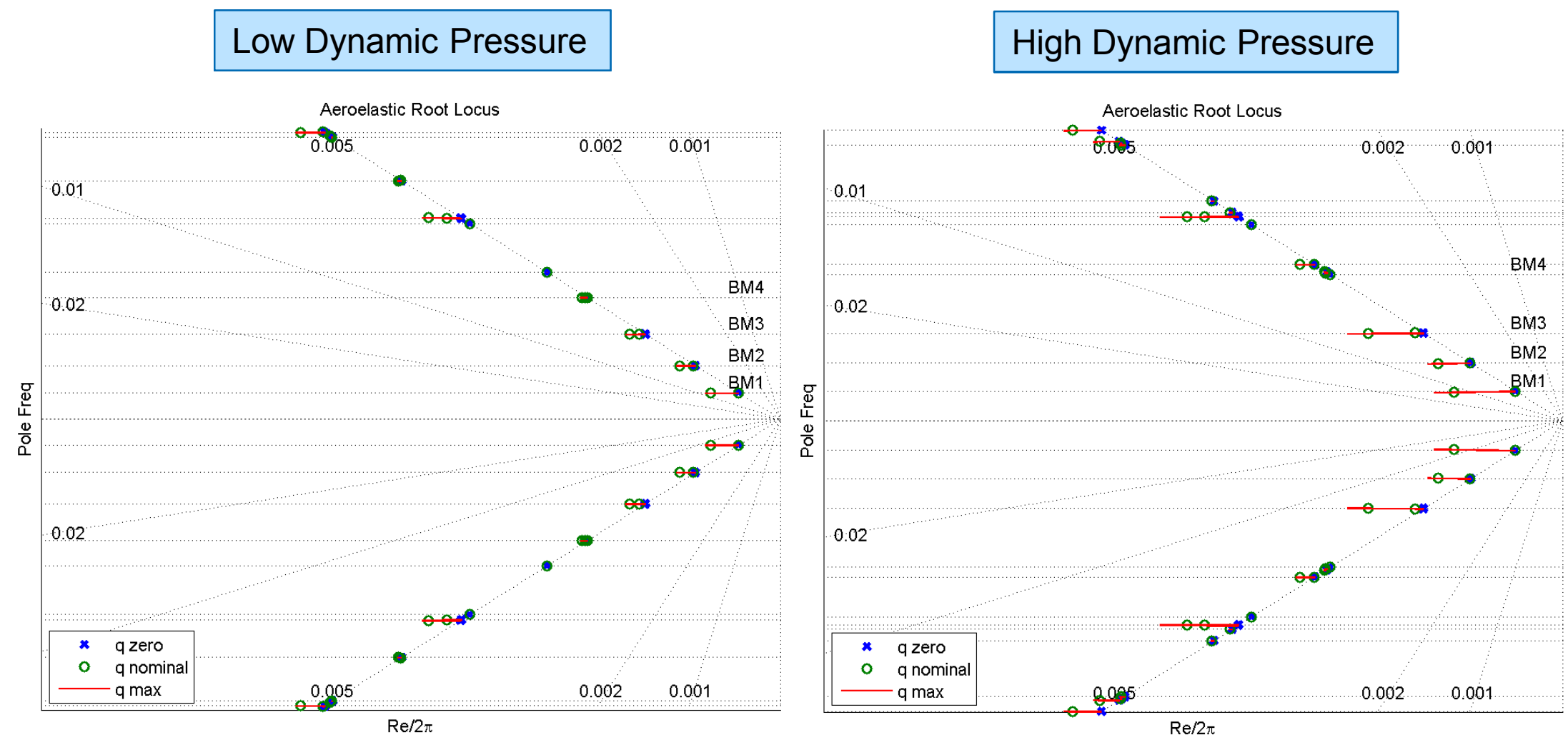


\section{Coupled Autopilot Stability Margin Analysis}

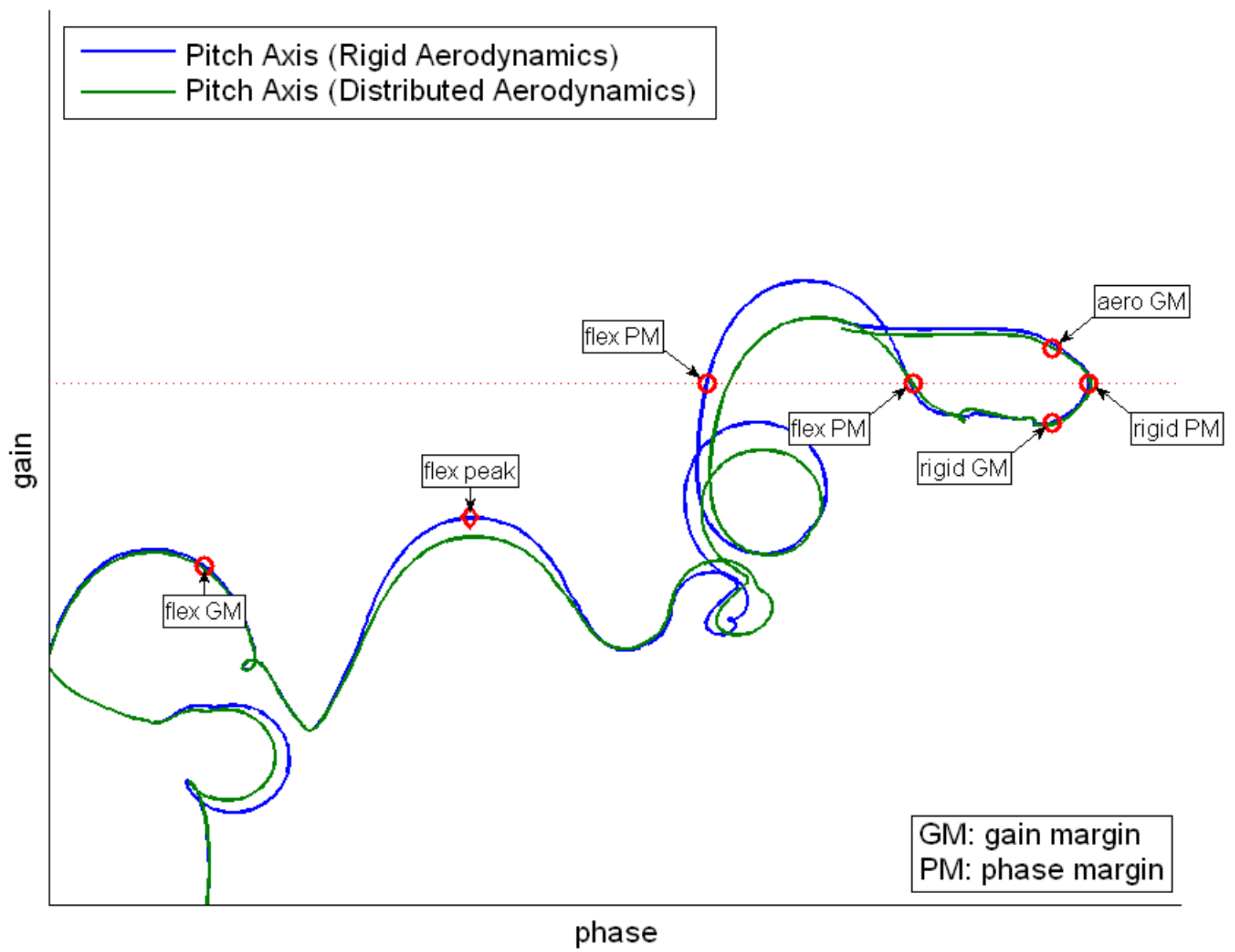




\section{Rigid Body Damping}

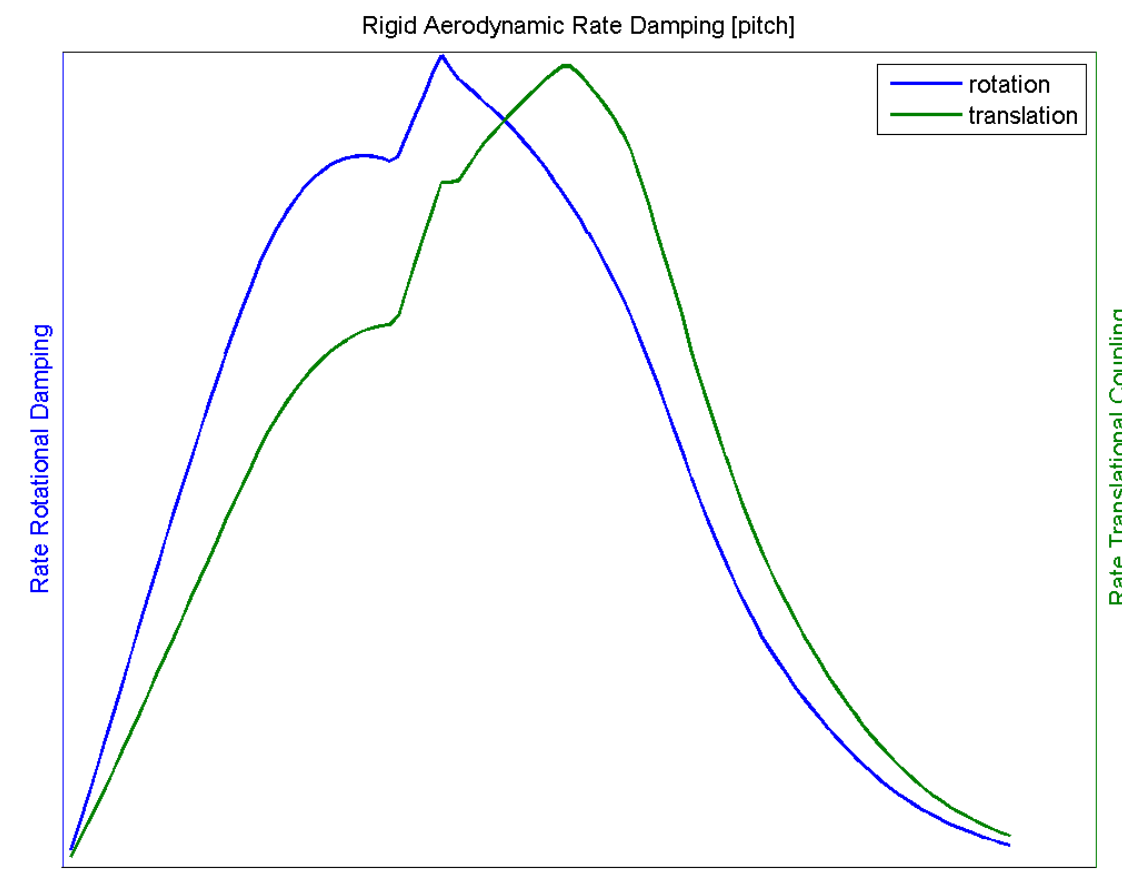

Flight Time

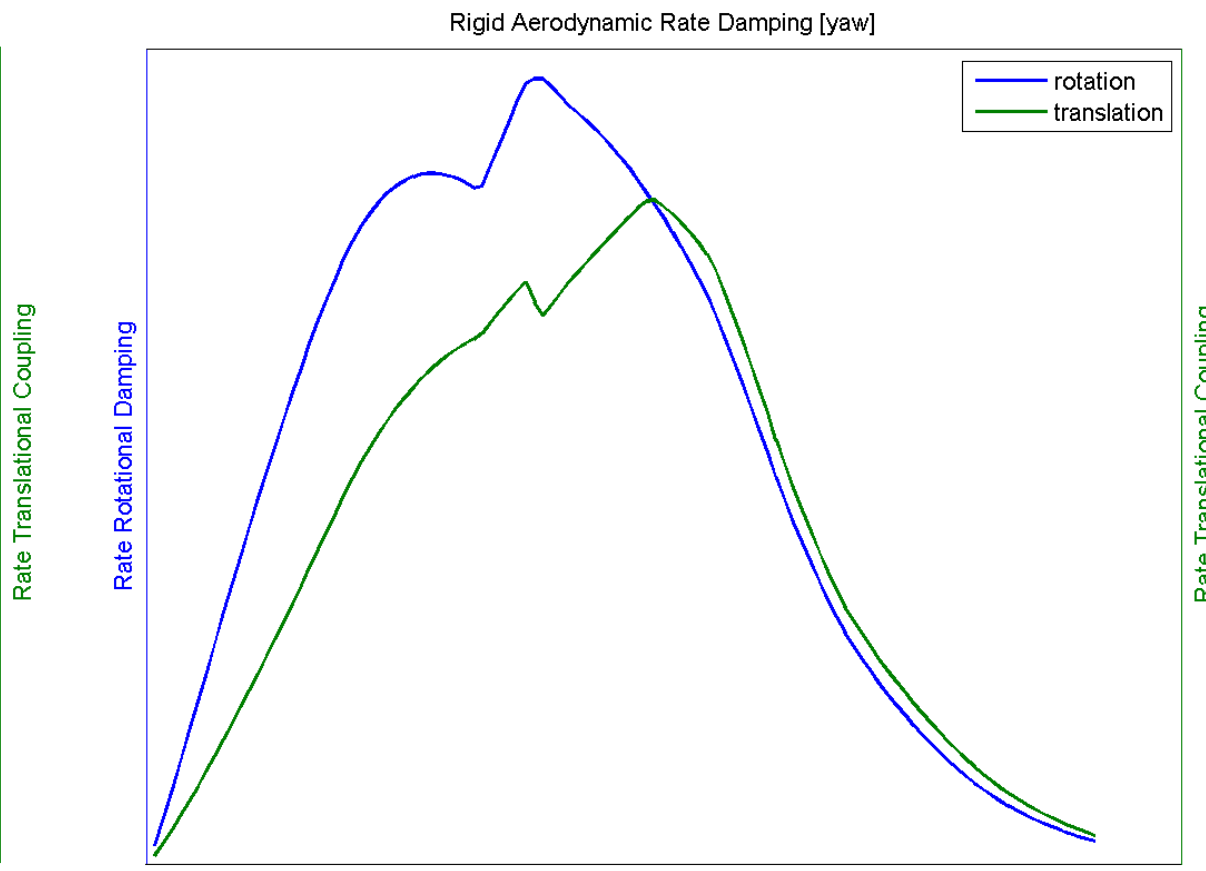

Flight Time 


\section{Coupled Dynamic Response Analysis}

FRACTAL gust response, distributed aero Scale Factor 20x / 40x / 20x (angle / flex / slosh )

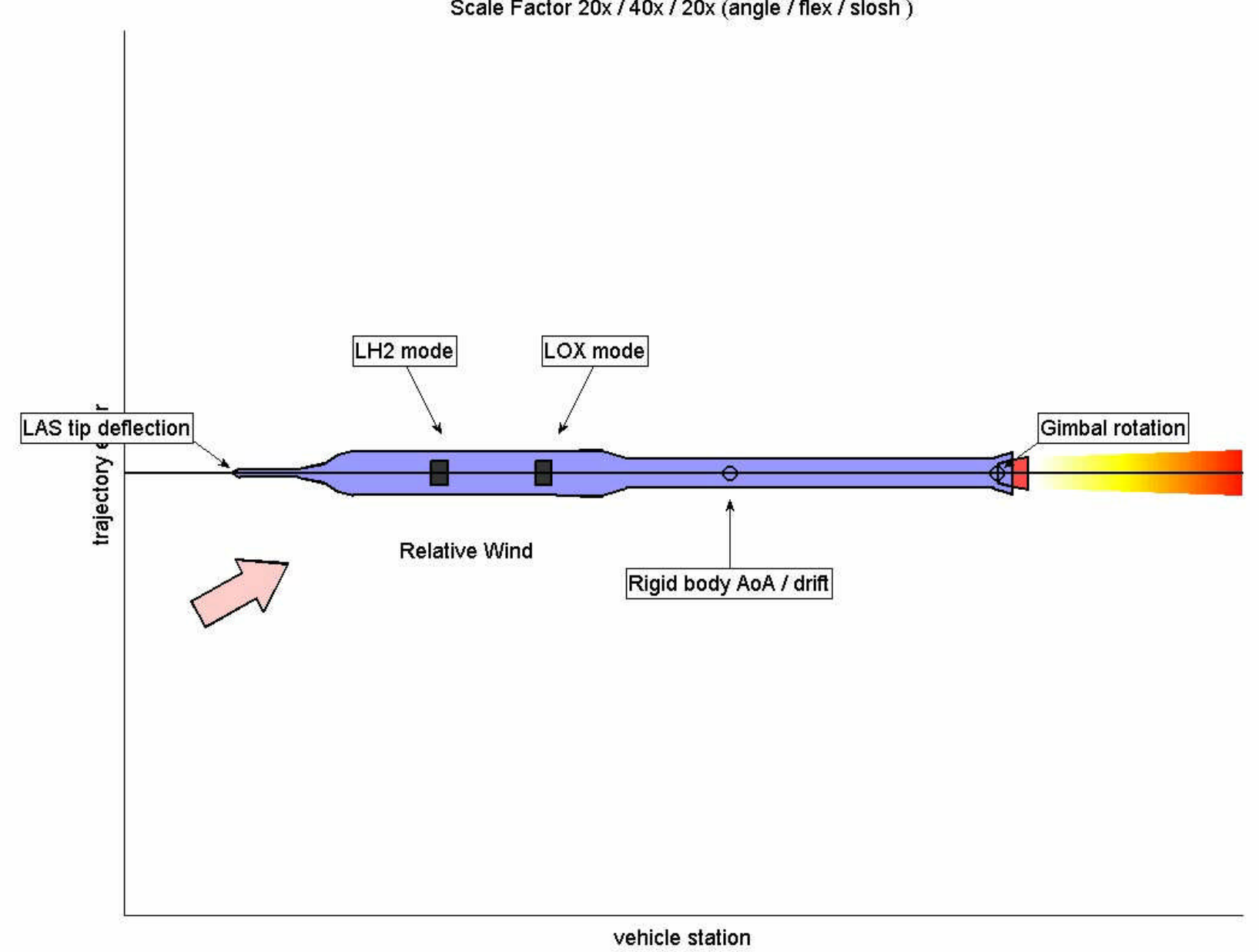

vehicle station 


\section{Summary and Discussion}

- A planar first-order linear aeroelastic model has been incorporated into the stability analysis dynamics engine previously presented

- Evaluation of aeroelastic coupling with other dynamics (autopilot, propellant slosh, nozzle and actuator) improves confidence in system stability

- Transient simulation allows for the evaluation of autopilot performance in the presence of external disturbances, e.g., gusts, steady-state wind, thrust vector bias, etc., in the presence of distributed loads

- Aeroelastic stability can be predicted in a single tool using standard metrics to determine susceptibility to flutter and divergence in the presence of closed-loop control

- The present approach is fast and computationally efficient

- Various other approaches exist for determining the coupled response; future work will compare these techniques

- Reduced models derived from coupled CFD-FEM analysis

- Time-domain nonlinear simulation

- Identification of structural response via flight test programmed test inputs (PTI) 